\title{
Near-infrared spectra of ISO selected Chamaeleon I young stellar objects ${ }^{\star}$
}

\author{
M. Gómez ${ }^{1}$ and P. Persi² \\ 1 Observatorio Astronómico de Córdoba, Laprida 854, 5000 Córdoba, Argentina \\ 2 Istituto Astrofisica Spaziale e Fisica Cosmica, CNR, Via del Fosso del Cavaliere 100, 00133 Roma, Italia
}

Received 15 January 2002 / Accepted 16 April 2002

\begin{abstract}
We present $0.95-2.5 \mu \mathrm{m}$ moderate $(R \sim 500)$ resolution spectra of 19 ISOCAM detected sources in the Chamaeleon I dark cloud. Thirteen of these stars are candidate very low mass members of the cloud proposed by Persi et al. (2000) on basis of the mid-IR color excess. The sample also includes a bona-fide young brown dwarf (Cha $\mathrm{H} \alpha$ 1), a transition - stellar/sub-stellar - object (Cha $\mathrm{H} \alpha 2$ ), one previously known $\mathrm{T}$ Tauri star (Sz 33) and three ISOCAM sources with no mid-IR excess. The spectra of the mid-IR color excess sources are relatively flat and featureless in this wavelength range. Both atomic and molecular lines (when in absorption) are partially veiled suggesting the presence of continuum emission from circumstellar dust. In addition some of the sources show Paschen and Brackett lines in emission. We apply the $2 \mu \mathrm{m}$ water vapor index defined by Wilking et al. (1999) to estimate spectral types. These stars have spectral types M0-8. We use Persi et al.'s stellar luminosity determinations, in combination with D'Antona \& Mazzitelli latest pre-main sequence evolutionary tracks, to estimate masses and ages. The ISOCAM detected mid-IR excess sources have sub-solar masses down to the H-burning limit and a median age of few $\times 10^{6} \mathrm{yr}$, in good agreement with the higher mass members of this cloud.
\end{abstract}

Key words. techniques: spectroscopic - stars: formation - stars: pre-main sequence - stars: Hertzsprung-Russell (HR) diagram - stars: low-mass, brown dwarfs - infrared: stars

\section{Introduction}

Recently Persi et al. (2000) have presented the results of the ISOCAM survey of the Chamaeleon I dark cloud. These observations cover $\sim 0.59 \mathrm{deg}^{2}$ of the cloud at 6.7 and $14.3 \mu \mathrm{m}$ to a detection limit of about $0.7 \mathrm{mJy}$ and $1.3 \mathrm{mJy}$, respectively. They detected 282 sources at $6.7 \mu \mathrm{m} ; 103$ of which were also found at $14.3 \mu \mathrm{m}$, including 74 previously known members of the cloud. These data, in combination with the DENIS near-IR observations (Cambrésy et al. 1998), have allowed Persi et al. (2000) to identify 34 very low luminosity $(L \sim 0.02$ $0.3 L_{\odot}$ ) candidate members of the Chamaeleon I dark cloud. These newly detected objects show significant midIR excess and fluxes at $6.7 \mu \mathrm{m}>2.5 \mathrm{mJy}$. They probably include very low mass stars in the cloud down to (and even below) the H-burning limit. We take advantage of the recent development of the near-IR spectroscopy to unveil the physical nature of thirteen of the ISOCAM proposed

\footnotetext{
Send offprint requests to: M. Gómez,

e-mail: mercedes@oac. uncor.edu

* Based on observations collected at the European Southern Observatory, Chile, (ESO proposal N.65.I-0054).
}

low mass members of the Chamaeleon I dark cloud. Most of our targets have $K \sim 11$ (see Table 1 ) and no optical counterparts on the Digitized Sky Survey. Thus the nearIR wavelengths provide a suitable range to study these sources.

$K$-band spectroscopic observations of young stellar objects in different star-forming regions at similar spectral resolution have been reported by several authors (Suto et al. 1989; Carr 1989; Carr 1990; Casali \& Matthews 1992; Casali \& Eiroa 1996; Greene \& Meyer 1995). In addition, Greene \& Lada (1996) have published an extensive atlas of near-IR spectra of about 100 young stellar objects. Wilking et al. (1999), Cushing et al. (2000), and Lucas et al. (2001) have used near-IR spectra to study candidates and bona-fide young brown dwarfs in the $\rho$ Ophiuchi and Orion clouds. These works provide a detailed reference to our targets as well as initial determinations of spectral features and characteristics usually present in the spectra of young stellar objects of different spectral energy distribution classes and masses.

We describe our observations and data reduction in Sect. 2. In Sect. 3 we analyze the spectral features of these sources and compare them with the near-IR spectroscopic 
characteristics of previously known young stellar objects. We apply the $2 \mu \mathrm{m}$ water vapor index defined by Wilking et al. (1999) to determine spectral types. We use Persi et al. (2000)'s stellar luminosity determinations, in combination with D'Antona \& Mazzitelli latest pre-main sequence evolutionary tracks, to estimate masses and ages for these stars. We discuss our results and conclude with a brief summary in Sect. 4 .

\section{Observations and data reduction}

We obtained $0.95-2.5 \mu \mathrm{m}$ spectra of 19 sources in the Chamaeleon I dark cloud. Thirteen of these sources are ISOCAM detected candidate young stars with significant mid-IR color excess. Three are previously known young members of the cloud (Cha $\mathrm{H} \alpha 1$, Cha $\mathrm{H} \alpha 2$, and $\mathrm{Sz} 33$ ) and other three are ISOCAM detected sources with no mid-IR excess. The 13 targets with mid-IR color excess were selected randomly among the 34 ISOCAM candidates proposed by Persi et al. (2000). Table 1 reports our sample, the infrared magnitudes and some derived photometric parameters obtained from the literature. We also observed three previously known pre-main sequence stars ( $\mathrm{Sz}$ 84, Sz 97, and $\mathrm{Sz} 105$ ) belonging to the Lupus starforming region. These known stars have M spectral types, optically determined, and will be used as comparison and reference to the ISOCAM sources.

The observations were carried out on April 27-28 2000 with the ESO NTT near-IR spectrograph/imaging camera SOFI (Son OF ISAAC). We observed our targets with the two low resolution grisms (red and blue) to roughly cover the $J H K$ bands on a Hawaii $\mathrm{HgCdTe} 1024 \times 1024$ detector at a plate scale of $0.292^{\prime \prime} /$ pix. The blue grism covers the spectral region between $0.95-1.63 \mu \mathrm{m}$ and the red grism the region between $1.53-2.52 \mu \mathrm{m}$. The corresponding spectral resolutions $(R=\lambda / \Delta \lambda)$ are 930 and 980 for a $0.6^{\prime \prime}$ slit. We used a $1^{\prime \prime}$ width and $290^{\prime \prime}$ length slit. This slit width provides an effective spectral resolution of approximately 560 and 590, blue and red grisms. The corresponding dispersions are $11.5 \AA /$ pix and $17.2 \AA /$ pix, respectively.

The integration time and the number of exposures per target were chosen according to the brightness of the individual sources and background contribution during each night. In most cases we obtained a total of 4 blue spectra of the same integration time, and 6 red spectra. Typical integration times are 80-400s for the blue and 180-240s for red grisms, respectively. The telescope was nodded 30$60^{\prime \prime}$ along the slit between consecutive positions following the usual ABBA pattern. This procedure corresponds to the "Nod Throw Along Slit" scheme as fully described in the SOFI Users Manual (Lidman et al. 2000).

In addition to the program sources we observed several atmospheric standards from the SOFI list of infrared spectroscopic standards (see the SOFI web page $^{1}$ ) for telluric absorption corrections. We selected two groups

\footnotetext{
1 http://www.ls.eso.org/lasilla/Telescopes/NEWNTT/ sofi/index.html.
}

of standards with similar airmass as our candidate sources and observed them periodically (i.e., every $\sim 2$ hours) during each night. These telluric stars comprise both late (G3-5) and early (O8-9) spectral type objects. We took 4 spectra per observations in both spectral regions. Total integration times ranged between 8 and $20 \mathrm{~s}$ for the two grisms. The stars were shifted along the slit direction by $60^{\prime \prime}$ between exposures, following the ABBA pattern.

We obtained multiple flat field images, in both grisms, with a dome screen, using incandescent lamps on and off. A xenon lamp, also taken on and off, each night provided the wavelength calibration for our data.

To reduce the data we used $\mathrm{IRAF}^{2}$. We subtracted one image from another (using pairs of nodded observations) to eliminate the background and sky contribution in first approximation. This subtraction automatically took care of dark current and bias level. We chose the images closer in time and position on the sky to perform this subtraction. We flat-fielded our data dividing by an appropriated normalized flat-field for each of the grisms. The flat-fields were created median-filtering multiple exposures in each grism. Bad pixels were replaced by linear interpolation from neighboring pixels.

We aligned individual exposures corresponding to each grism and target and combined the corresponding frames into one blue and one red image per object. To do this alignment as accurate as possible we traced each spectrum as describe below and determine a precise center. Then we shifted all data, corresponding to each target and grism, to a common position and co-added them.

We used the twodspec task APALL to trace and extract the spectra along a 12 pixel wide aperture on the co-added blue and red images. A further sky subtraction was done by fitting a polynomial to the regions on either side of the aperture. Residual sky contributions were corrected reasonably well by this procedure. A non linear low order fit to the lines in the xenon lamp was used to wavelength calibrate the spectra. We verified our wavelength solution using the positions of well known telluric lines as well as some $\mathrm{H}$ lines in the spectra of the standard stars.

We removed telluric features from our data dividing the program spectra by the atmospheric standard spectra. At the spectral resolution used $(R \sim 500-600)$ the O89 stars provided quasi featureless spectra in all the spectral range covered by the two grisms. The only spectral lines present are strong $\mathrm{H}$ and $\mathrm{He}$ lines. We used the onedspec task SPLOT to remove $\mathrm{H}$ (Paschen and Brackett) and He (I and II) lines from the spectra of the O stars, interpolating across each line. G5-3 stars, on the contrary, display weak Paschen and Brackett series but contain atomic (metallic) lines in addition to some molecular features. The spectra of these solar type stars were used with no modifications (i.e., no lines were removed).

\footnotetext{
${ }^{2}$ IRAF is distributed by the National Optical Astronomy Observatory, which is operated by the Association of Universities for Research in Astronomy, Inc. under contract to the National Science Foundation.
} 
Table 1. Compiled photometric magnitudes and derived parameters for the nineteen ISO sources spectroscopically observed.

\begin{tabular}{|c|c|c|c|c|c|c|c|c|c|c|c|}
\hline Name & $\overline{I^{\mathrm{a}}}$ & $\overline{K^{\mathrm{b}}}$ & $H-K^{\mathrm{b}}$ & $\bar{J} J-H^{\mathrm{b}}$ & $\overline{L^{\mathrm{c}}}$ & mag $6.7^{\mathrm{d}}$ & mag $14.3^{\mathrm{d}}$ & $\overline{A_{J}^{\mathrm{d}}}$ & 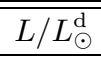 & $\overline{\alpha_{\text {IR }}{ }^{\mathrm{d}}}$ & Other ID \\
\hline ISO-ChaI 79 & & 12.39 & 1.23 & 1.78 & & 9.83 & 6.39 & $2.4^{*}$ & 0.07 & -0.8 & \\
\hline ISO-ChaI 95 & 16.58 & 12.31 & 0.45 & 0.73 & & 10.03 & 6.20 & 0.3 & 0.02 & & Cha $\mathrm{H} \alpha 1^{\mathrm{e}}$ \\
\hline ISO-ChaI 98 & 17.61 & 11.89 & 0.71 & 1.38 & & 9.45 & 6.47 & 2.0 & 0.07 & -1.2 & \\
\hline ISO-ChaI 111 & 15.26 & 10.65 & 0.57 & 1.09 & 9.94 & 8.62 & 5.46 & 1.3 & 0.08 & & Cha $\mathrm{H} \alpha 2^{\mathrm{e}}$ \\
\hline ISO-ChaI 138 & 16.74 & 13.05 & 0.41 & 0.69 & & 9.13 & 5.77 & 1.3 & 0.03 & -0.2 & \\
\hline ISO-ChaI 143 & 15.43 & 11.09 & 0.55 & 1.09 & 10.67 & 9.11 & 5.84 & 1.3 & 0.11 & -1.2 & \\
\hline ISO-ChaI 154 & 14.42 & 11.37 & 0.33 & 1.01 & & 10.22 & & 0.3 & 0.04 & & \\
\hline ISO-ChaI 158 & 13.56 & 11.25 & 0.29 & 0.70 & & 11.34 & & & & & \\
\hline ISO-ChaI 209 & & 12.22 & 1.24 & 2.00 & & 10.03 & 6.77 & $3.0^{*}$ & 0.06 & -1.2 & DENIS-P J1109.8-7714 \\
\hline ISO-ChaI 220 & 18.11 & 12.48 & 0.85 & 1.25 & & 10.28 & 6.82 & 2.0 & 0.05 & -1.1 & \\
\hline ISO-ChaI 224 & 13.99 & 9.10 & 0.74 & 1.35 & 8.22 & 7.45 & 4.10 & 1.4 & 0.48 & & $\mathrm{Sz} 33^{\mathrm{f}}$ \\
\hline ISO-ChaI 225 & 17.12 & 12.75 & 0.98 & 1.11 & & 9.08 & 5.81 & 0.8 & 0.02 & -0.2 & \\
\hline ISO-ChaI 235 & 17.93 & 11.18 & 0.90 & 1.49 & & 10.94 & & 2.3 & 0.14 & -1.1 & \\
\hline ISO-ChaI 238 & 17.58 & 12.19 & 0.83 & 1.42 & & 9.10 & 5.79 & & & & \\
\hline ISO-ChaI 239 & 15.45 & 10.47 & 0.56 & 1.47 & 9.85 & 9.85 & & & & & \\
\hline ISO-ChaI 250 & 15.57 & 10.71 & 0.60 & 1.52 & & 8.84 & 7.03 & 1.6 & 0.20 & -1.9 & \\
\hline ISO-ChaI 252 & 17.11 & 12.29 & 0.60 & 1.12 & & 10.24 & 6.80 & 1.7 & 0.06 & -1.1 & \\
\hline ISO-ChaI 256 & 17.51 & 10.78 & 0.93 & 1.62 & & 8.13 & 4.69 & 2.4 & 0.17 & -0.8 & \\
\hline ISO-ChaI 282 & 15.52 & 11.88 & 0.61 & 1.00 & & 9.89 & 6.60 & 1.0 & 0.06 & -1.4 & \\
\hline
\end{tabular}

a Cambrésy et al. (1998).

b Gómez \& Kenyon (2001).

c Kenyon \& Gómez (2001).

d Persi et al. (2000).

e Comerón et al. (1998).

f Schwartz (1977).

*Note: Calculated from $A_{J}=2.63\left[(J-H)-(J-H)_{\mathrm{o}}\right]$, adopting $(J-H)_{\mathrm{o}}=0.85$ (see Persi et al. 2001).

For our ISOCAM targets we obtained two telluric corrected spectra using as telluric calibrators the early and the solar type atmospheric standard spectra. These standards differed from the science targets by $<0.2$ in airmass. In addition, the time interval between the observations of the pair target-standard was typically $<1$ hour.

The division by the 08-9 standard canceled out telluric features in the science spectrum reasonable well but introduced the inverse of the atmospheric standard in the corrected spectrum. We recovered the true spectral shape multiplying the resultant spectrum by a Planck function at the temperature corresponding to the atmospheric star. We also used the atmosphere models of Kurucz ${ }^{3}$ and the library of spectra from Pickles (1998) for the appropriate $T_{\text {eff }}$. These procedures gave essentially the same result.

We used the normalized solar spectrum ${ }^{4}$, convolved to the SOFI blue and red grism resolutions, to multiply the second set of science spectra, the ones divided by the G3-5 telluric standards. We used a Gaussian profile to smooth the solar spectrum and a Planck function at the tempera-

\footnotetext{
3 Available at http://cfaku5.harvard.edu/grids.html.

4 NSO/Kitt Peak FTS data used here were produced by $\mathrm{NSF} / \mathrm{NOAO}$.
}

ture of the telluric standard to reproduce the corresponding stellar continuum. Kurucz models and Pickles spectra for the telluric continuum, combined with the appropriate convolved normalized solar spectrum, produced basically identical corrected spectra for our science targets.

We finally combined the blue and the red grism spectra for each object and eliminated regions of deep atmospheric absorptions from our analysis as not satisfactory corrections were obtained in these regions. In addition we also trimmed out wavelengths between $0.95-0.978 \mu \mathrm{m}$ as edge effects and a poor $S / N$ ratio were present. The useful spectral ranges are: 9780-11000 $\AA, 11600-13300 \AA$, 14600-17800 , and 20500-25000 . The resultant telluric corrected spectra using both set of standards were essentially identical and display the spectral shapes and features of the science targets. In Sect. 3.2 we chose to show the set of science spectra obtained applying the G35 standards as telluric corrector.

\section{Data analysis and results}

\subsection{The near-IR $J-H$ vs. $H-K$ diagram}

Table 1 compiles photometry and derived parameters for the 19 sources reported in this paper from the literature. 
Figure 1 shows the positions of these stars in the nearIR color-color plot. The dotted symbols and the crosses represent stars in our sample with no mid-IR excess and previously identified members of the cloud, respectively. The big stars correspond to objects with mid-IR excess. Superposed on this diagram are the loci of unreddened main sequence dwarfs (solid line, Bessell \& Brett 1988) and CTTS - classical T Tauri stars - (long-dashed line, Meyer et al. 1997). The reddening band (dotted line, Gómez \& Kenyon 2001) and the length of the reddening vector (Rieke \& Lebofsky 1985) are also indicated.

Taking into account typical photometric errors for these sources $(\sim 0.03 \mathrm{mag}$ for $K=11$, see Gómez \& Kenyon 2001), we found that approximately $70 \%$ (9 out of 13) of the stars selected on basis of the mid-IR excess show no significant near-IR excess emission. Persi et al. (2000) classified these ISOCAM detected stars as Class II members of the Chamaeleon I dark cloud using the infrared spectral index $\left(\alpha_{\mathrm{IR}}=\mathrm{d} \log \left(\lambda F_{\lambda} / \mathrm{d} \log (\lambda)\right)\right.$, computed from 2.2 to $14.3 \mu \mathrm{m}$. They typically have $-1.6<\alpha_{\mathrm{IR}}<0.3$, corresponding to Class II objects (see Table 1 ). The only exception is ISO-ChaI 250 with $\alpha_{\mathrm{IR}}=-1.9$, identified as a Class II-III member of the cloud by Persi et al. (2000). The CTTS or the Class II objects usually have detectable near-IR excesses. However, a significant fraction of the bona-fide CTTS in the Taurus (e.g., Strom et al. 1993; Kenyon \& Hartmann 1995) and in the Chamaeleon I (e.g., Gauvin \& Strom 1992; Comerón et al. 2000) star forming regions shows no measurable near-IR excesses. Therefore, mid-IR observations are a very powerful tool to discriminate young stellar objects from background stars (cf. Persi et al. 2000).

\subsection{The near-IR spectra}

Figures 2-7 show the infrared spectra of the 19 sources reported in this paper. Prominent atomic and molecular features in the $0.98-2.45 \mu \mathrm{m}$ range for late type stars (i.e., spectral types later than G0) are labeled (Wallace et al. 2000; Meyer et al. 1998; Wallace \& Hinkle 1997). Table 2 lists equivalent widths for the some of the strongest lines. We estimate an uncertainty of $\sim 1-2 \AA$ in the $\mathrm{Pa} \beta, \mathrm{Br} \gamma$, $\mathrm{Na}$ I doublet $(2.206$ and $2.209 \mu \mathrm{m})$, and Ca I triplet (2.261, 2.263 , and $2.266 \mu \mathrm{m}$ ) equivalent widths. For the combine $\mathrm{CO} \nu=0-2$ and $2-4$ bands the uncertainty in our measurements is $\sim 3 \AA$. Stars in Table 2 have, on average, similar or slightly smaller equivalent widths than $M$ type standards (Kleinmann \& Hall 1986; Wallace et al. 2000), with exception of few sources (such as ISO-ChaI 79, ISOChaI 143, and ISO-ChaI 225) with no absorption lines present at the spectral resolution used.

\subsubsection{ISO detected sources with no mid-IR excess}

Near-IR spectra of three ISOCAM detected sources without near- and mid-IR excesses (ISO-ChaI 158, ISOChaI 238, and ISO-ChaI 239) are illustrated in Fig. 2.
Table 2. Equivalent widths (in $\AA$ ) for the nineteen ISO sources analyzed*.

\begin{tabular}{|c|c|c|c|c|c|}
\hline \multirow[t]{2}{*}{ Name } & $\overline{\mathrm{Pa} \beta}$ & $\overline{\mathrm{Br} \gamma}$ & $\mathrm{NaI^{ \textrm {a } }}$ & $\overline{\mathrm{Ca} \mathrm{I}}{ }^{\mathrm{b}}$ & $\mathrm{CO}^{\mathrm{c}}$ \\
\hline & $1.28 \mu \mathrm{m}$ & $2.17 \mu \mathrm{m}$ & $2.21 \mu \mathrm{m}$ & $2.26 \mu \mathrm{m}$ & $2.23-2.38 \mu \mathrm{m}$ \\
\hline \multicolumn{6}{|l|}{ ISO-ChaI 79} \\
\hline ISO-ChaI 95 & 0.7 & 4.5 & 4.8 & 2.0 & 37.5 \\
\hline ISO-ChaI 98 & -2.9 & -3.6 & & 3.0 & 16.5 \\
\hline ISO-ChaI 111 & 1.4 & 3.9 & & & 11.9 \\
\hline ISO-ChaI 138 & -1.5 & -0.7 & 4.7 & 3.4 & 27.2 \\
\hline ISO-ChaI 143 & -3.2 & & & & \\
\hline ISO-ChaI 154 & 2.3 & & & & \\
\hline ISO-ChaI 158 & 1.9 & 2.9 & & & 13.7 \\
\hline ISO-ChaI 209 & 5.1 & 11.2 & & & 31.7 \\
\hline ISO-ChaI 220 & 2.9 & -4.6 & & & 5.5 \\
\hline ISO-ChaI 224 & -3.0 & -1.5 & 2.5 & 2.3 & 18.9 \\
\hline \multicolumn{6}{|l|}{ ISO-ChaI 225} \\
\hline ISO-ChaI 235 & 1.3 & 5.0 & & 4.5 & 20.0 \\
\hline ISO-ChaI 238 & & & & & 40.5 \\
\hline ISO-ChaI 239 & 2.0 & 3.2 & 2.3 & 1.4 & 43.8 \\
\hline ISO-ChaI 250 & 3.2 & & 4.8 & 4.8 & 34.6 \\
\hline ISO-ChaI 252 & -27.2 & & 7.7 & & 31.7 \\
\hline ISO-ChaI 256 & -5.6 & & 2.4 & 4.1 & 28.0 \\
\hline ISO-ChaI 282 & 0.9 & & & & 37.0 \\
\hline
\end{tabular}

${ }^{\text {a }} \mathrm{Na}$ I doublet $(2.206$ and $2.209 \mu \mathrm{m})$.

b Ca I triplet $(2.261,2.263$, and $2.266 \mu \mathrm{m})$.

${ }^{c} \mathrm{CO} \nu=0-2$ and 2-4 bands.

${ }^{*}$ Note: Positive values indicate absorptions and negative values correspond to emissions. Missing equivalent widths indicate that the corresponding feature was not detected either in absorption or emission at the resolution used $(R \sim 950)$.

The spectra of these targets show no sign of activity, such as emission lines (at the spectral resolution used), characteristic of young stars. As we will derive in Sect. 3.3.1 these stars have M type spectral types and are likely background objects, seen at the near-IR wavelengths through the cloud.

\subsubsection{Previously known young stellar objects}

Figure 3 shows our near-IR spectra of three previously known young stellar objects in the Chamaeleon I dark cloud: ISO-ChaI 95 (Cha $\mathrm{H} \alpha$ 1), ISO-ChaI 111 (Cha $\mathrm{H} \alpha 2$ ), ISO-ChaI 224 ( $\mathrm{Sz} 33)$. Cha $\mathrm{H} \alpha 1$ ( $M \sim$ $\left.0.05 M_{\odot}\right)$ and Cha $\mathrm{H} \alpha 2\left(M \sim 0.08 M_{\odot}\right)$ are a bona-fide brown dwarf and a transition (stellar/sub-stellar) object of the cloud (Comerón et al. 2000). Comerón et al. (1998) measured $E W(\mathrm{H} \alpha)$ of 59 and $39 \AA$ for Cha $\mathrm{H} \alpha 1$ and Cha $\mathrm{H} \alpha 2$, respectively, corresponding to the $E W(\mathrm{H} \alpha)$ associated with the solar mass classical $\mathrm{T}$ Tauri stars (CTTS). They also estimated optical M7.5-8 and M6 spectral types for these sub-stellar or quasi sub-stellar objects. Neuhäuser \& Comerón (1998) detected Li $6707 \AA$ in higher resolution spectra assuring the pre-main sequence status of these objects.

Comerón et al. (2000) obtained $H K$ band spectra for a sample of 11 extremely low mass members of 


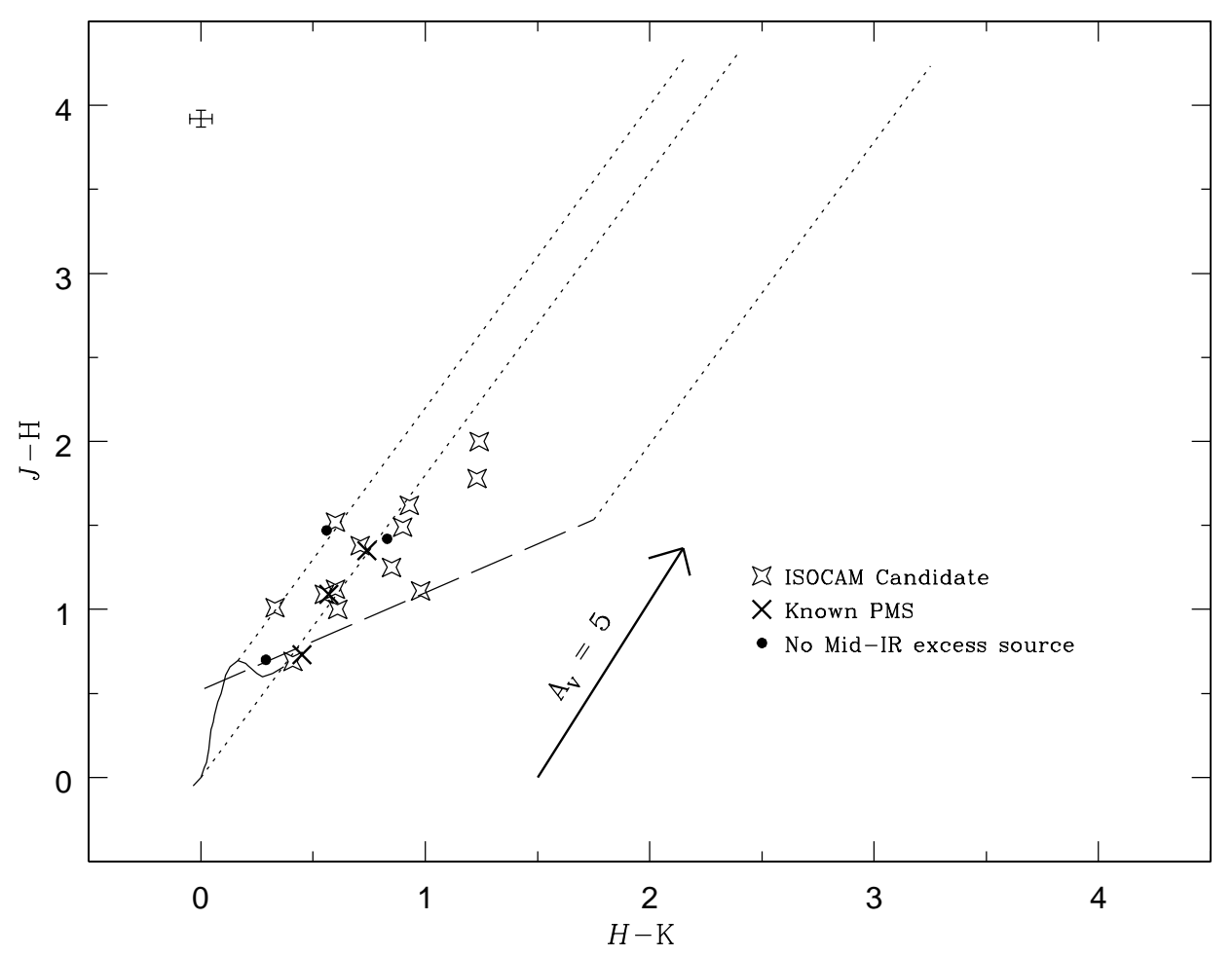

Fig. 1. Near-IR color-color diagram for the ISOCAM sources listed in Table 1. The solid and long-dashed lines indicate the loci of unreddened main sequence dwarfs (Bessell \& Brett 1988) and CTTS (Meyer et al. 1997). The dotted lines define the reddening band, corresponding to a reddening vector $E(J-H) / E(H-K)=1.80$ (Gómez \& Kenyon 2001). The arrow indicates an $A_{V}=5 \mathrm{mag}$ (Rieke \& Lebofsky 1985). Typical photometric errors are displayed in the upper left corner.

the Chamaeleon I dark cloud, including Cha $\mathrm{H} \alpha 1$ and Cha $\mathrm{H} \alpha 2$. These objects have spectral types, optically determined, within a very narrow range (M 6-8). These authors published averaged near-IR spectra for stars with basically identically optical spectral types. In this manner, the three averaged near-IR spectra (representative of objects with M6, M 7, and M 8 sub-types) have a better $S / N$ ratio than the individual spectra of each of the 11 sources. Cha $\mathrm{H} \alpha 1$ and Cha $\mathrm{H} \alpha 2$ are included in the $\mathrm{M} 8$ and M 6 groups, respectively. An individual spectrum corresponding to Cha $\mathrm{H} \alpha 1$ was also published by Neuhäuser \& Comerón (1998).

Our spectra for ISO-ChaI 95 and ISO-ChaI 111 agree well with the averaged spectra or with the individual spectrum in the case of ISO-ChaI 95 of previous works. We additionally observed the $J$ band as shown in Fig. 3. Both objects show a decreasing spectral shape toward longer wavelengths. No prominent spectral features appear in all the wavelength covered. In particular $\mathrm{H}$ (Paschen, and Brackett) lines are not in emission, at this spectral resolution.

Lawson et al. (1996) derived a mass of $\sim 0.3-0.4 M_{\odot}$ for $\mathrm{Sz} 33$ (CHX15b=CHXR41; Feigelson \& Kirss 1989; Feigelson et al. 1993). This sub-solar mass young star represents the only previously known $\mathrm{T}$ Tauri star in our sample. It has been classified as a WTTS (weak T Tauri star) member of the cloud (Gauvin \& Strom 1992; Lawson et al. 1996). However, Lawson et al. (1996) casted some doubts upon the WTTS status of this object. In fact, $\mathrm{Sz} 33$ has near-IR spectroscopic characteristics more frequently found associated with the Class II objects (CTTS) than with the Class III stars (WTTS; see Greene \& Lada 1996). In particular both Paschen 5 and $6(\mathrm{~Pa} \beta, \mathrm{Pa} \delta)$ and Brackett $7(\mathrm{Br} \gamma)$ are in emission whereas the spectral lines are moderately veiled at our resolution (see Tables 2 and 4). The spectral shape of Sz 33 strongly decreases with increasing wavelength.

\subsubsection{ISO candidate young stellar objects}

Our near-IR spectra for thirteen of the new young stellar objects found by the ISOCAM survey in the Chamaeleon I dark cloud are shown in Figs. 4-7. As a group these sources show a variety of spectral shapes. Several stars have fairly constant shapes over the $0.95-2.45 \mu \mathrm{m}$ range. Other sources display a smoothly decreasing spectral shape with increasing wavelength or have a turnover around 1.5$1.6 \mu \mathrm{m}$. Absorption lines are partially or complete veiled (see Tables 2 and 4). ISO-ChaI 79 and ISO-ChaI 225 show strongly veiled spectra. These stars, in addition to ISOChaI 209 and ISO-ChaI 220, also display the largest nearIR excesses in Fig. 1 and are thus probably surrounding by significant amount of circumstellar material. Some objects (ISO-ChaI 252, ISO-ChaI 98, ISO-ChaI 143, ISOChaI 220, and ISO-ChaI 256, see Table 2) have H lines 


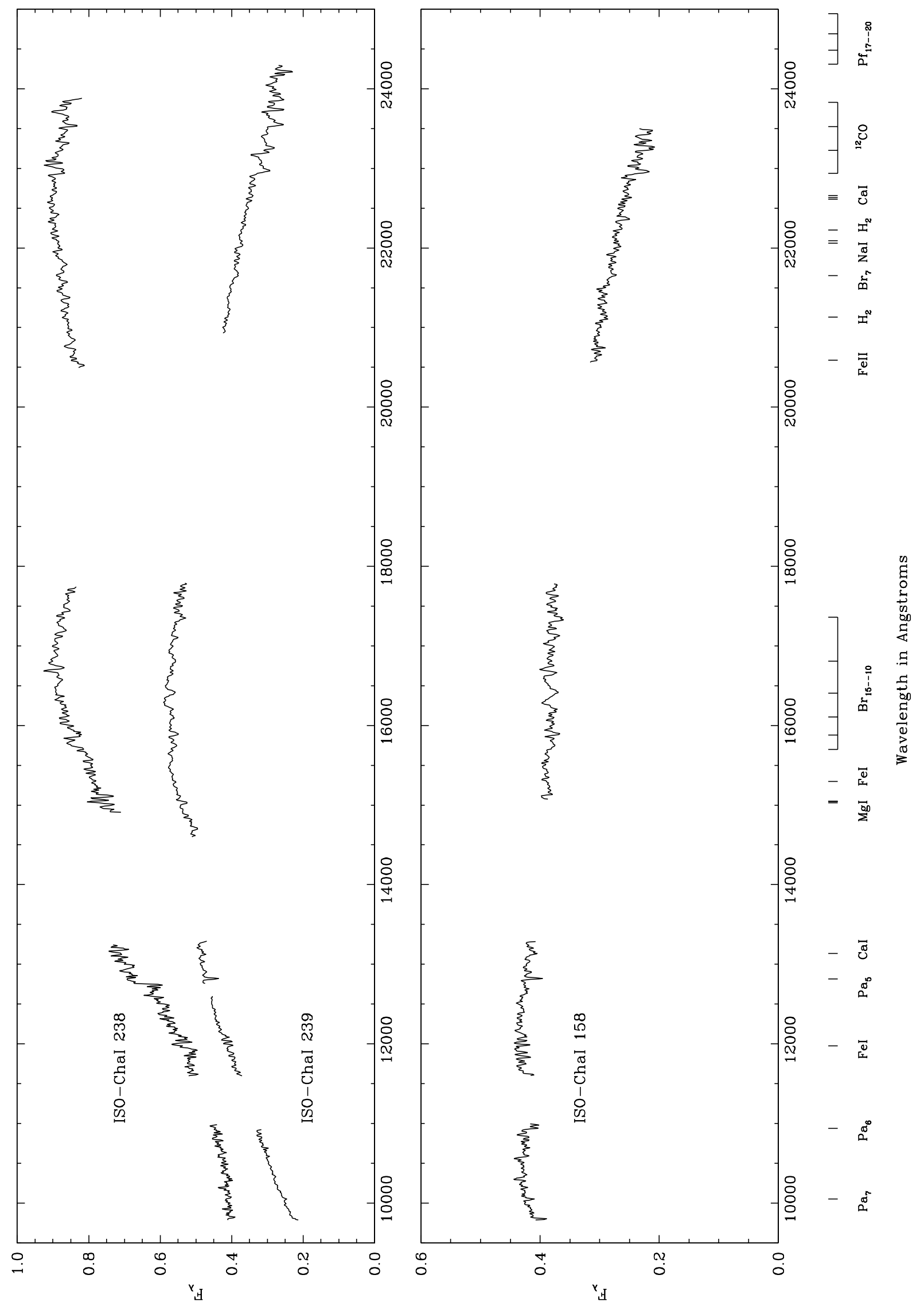

Fig. 2. $J H K$ band spectra of ISOCAM detected sources in the Chamaeleon I dark cloud with no mid-IR excess. The vertical scale $\left(F_{\lambda}\right)$ corresponding to ISO-ChaI 238 is offset by 0.82 with respect to ISO-ChaI 239. Prominent atomic and molecular features in the $0.98-2.45 \mu \mathrm{m}$ range are labeled. 


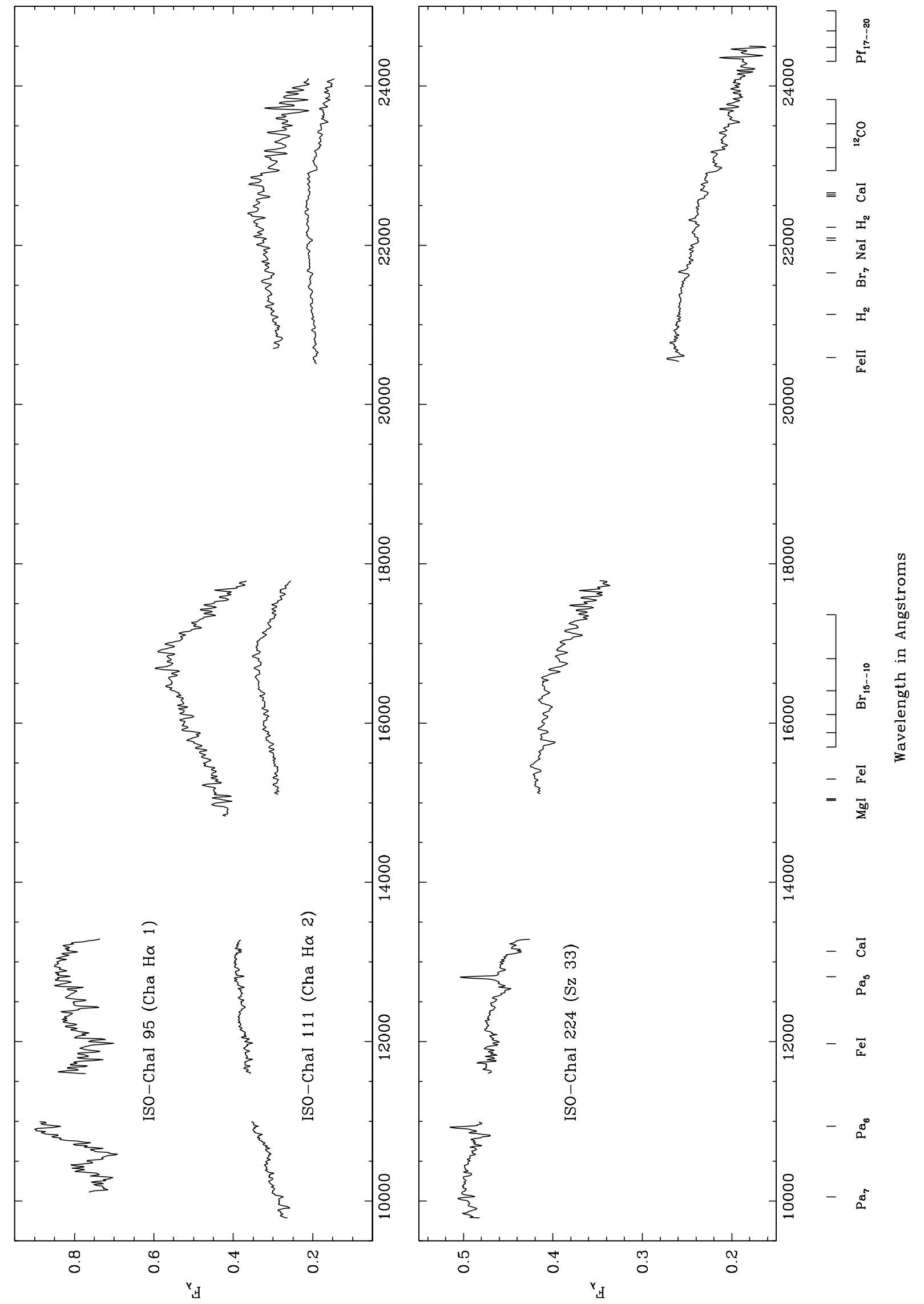

Fig. 3. $J H K$ band spectra of three previously known young stellar objects in the Chamaeleon I dark cloud. Prominent atomic and molecular features in the $0.98-2.45 \mu \mathrm{m}$ range are labeled. 
in emission, also indicating the presence of disks as we discuss in Sect. 3.4.

These near-IR spectral characteristics are common among the Class II population of other well known starforming regions such as $\rho$ Ophiuchi and Taurus (Greene \& Lada 1996). In general the Class II objects show a wider range of variation in different spectral features such as veiling, spectral shape or emission line intensities. For Class I and Class III objects these parameters vary over a smaller range or, in other words, the near-IR spectroscopic characteristics of Class I and Class III objects are more similar within each group than among the Class II objects (see Greene \& Lada 1996 's atlas).

For ISO-ChaI 79 we have eliminated the spectral region between $13000 \AA$ and $20000 \AA$. This ISOCAM candidate is one of our faintest targets (see Table 1). The background contribution was relatively high for this star making this correction unreliable in this spectral range. ISO-ChaI 79 was also observed under relatively unfavorable weather conditions resulting in a poor correction of the deep water atmospheric features and noisy spectrum.

ISO-ChaI 154 displays a continuous decreasing spectrum and practically no evidence of veiling (see Table 4) and no emission lines, at the spectral resolution we used. This spectral behavior is similar to many Class III or WTTS (Greene \& Lada 1996). An optical (4500-8500 A) low resolution spectrum of this object, obtained at the CASLEO (San Juan, Argentina) with the REOSC spectrograph (DS mode) at the 2.15-m tel., shows no $\mathrm{H} \alpha$ in emission. However, the modest $S / N$ ratio of these data does not allow us to discard the presence of $\mathrm{H} \alpha$ in emission with a relatively small equivalent width (i.e., $E W(\mathrm{H} \alpha)<10 \AA)$. ISO-ChaI 154 might be a Class III member of the cloud.

\subsection{Spectral types and mass determinations for the ISO selected stars}

\subsubsection{The water vapor index $Q$}

Figures 2-7 show that even outside regions of atmospheric water vapor absorptions, the spectra of these objects have broad stellar $\mathrm{H}_{2} \mathrm{O}$ absorptions characteristic of low temperature objects. The strength of these bands are extremely sensitive to the spectral types for $\mathrm{M}$ dwarfs. Thus the stellar water features can be used to estimate spectral types for our targets. Wilking et al. (1999) have derived the index $Q$ that measures the strength of the $\mathrm{H}_{2} \mathrm{O}$ bands around $2 \mu \mathrm{m}$. This index, analogous to the index $Q$ used by Johnson \& Morgan (1953) in the $U B V$ system, is reddening independent. Wilking et al. (1999) defined the $2 \mu \mathrm{m}$ index $Q$ by the average values of relative flux density calculated in three narrow bands, $F 1(2.07-2.13 \mu \mathrm{m})$, $F 2(2.267-2.285 \mu \mathrm{m})$, and $F 3(2.40-2.50 \mu \mathrm{m})$, as

$Q=(F 1 / F 2)(F 3 / F 3)^{1.22}$.

In this definition these authors have adopted the reddening law from Rieke \& Lebofsky (1985), $A_{\lambda} \propto \lambda^{-1.47}$.
Table 3. Spectral types derived from the $Q$ index using G5 and $\mathrm{O} 8$ telluric standards for previously known objects.

\begin{tabular}{lcccl}
\hline \hline Name & G5 Tel. Std. O8 Tel. Std. & Optical & Other ID \\
\hline Cha H $\alpha 1$ & M8 & M9 & M7.5 & ISO-ChaI 95 \\
Cha H $\alpha 2$ & M5.5 & M6.5 & M6.5 & ISO-ChaI 111 \\
Sz 33 & M0 & M2 & M0 ${ }^{\text {b }}$ & ISO-ChaI 224 \\
Sz 84 & M5 & M4 & M4.5 & \\
Sz 97 & M3.5 & M2 & M $^{\mathrm{c}}$ & \\
Sz 105 & M5 & M3.5 & M4 & \\
\hline
\end{tabular}

${ }^{a}$ Comerón et al. (1998).

b Gauvin \& Strom (1992).

${ }^{\mathrm{c}}$ Hughes et al. (1994).

Wilking et al. (1999) applied this index to estimate spectral types for a sample of brown dwarfs in the $\rho$ Ophiuchi cloud. The same index, $Q$, has been used by Cushing et al. (2000) to provide spectral types for additional objects in the same cloud.

Both Wilking et al. (1999) and Cushing et al. (2000) have obtained linear relations to determine the M sub-type as function of $Q$ using $\mathrm{M}$ dwarf standards with optically known spectral types (see Eq. (2) in Wilking et al. 1999; Cushing et al. 2000). These relations have been derived from standards whose spectra were telluric corrected using A0V stars but whose continuum shapes were not restored.

We applied Wilking et al. (1999) and Cushing et al. (2000) calibrations to obtain spectral types for our targets. We used our atmospheric telluric divided spectra with no correction for the slope of the standard spectrum keeping in mind that our atmospheric telluric standards have G3-5 and 08-9 spectral types and then differ from those used by these authors. However, spectral types obtained using both our sets of standards differ by less than 1.5 sub-types. This difference is comparable to the accuracy expected by Wilking et al. (1999) and Cushing et al. (2000) for their calibrations. As an additional check we confronted spectral types derived using both our G3-5 and O8-9 spectral type standards for a sample of 6 previously known objects. In Table 3 we list these stars together with spectral types derived using our G5 and O8 telluric standards and the optical spectral types obtained from the literature. Our determinations agree with the optical types within roughly 1.5 types.

Table 4 gives spectral types for the ISOCAM detected candidate low mass members of the Chamaeleon I dark cloud, obtained using the G5 telluric standards. In general, spectral types derived using this set of standards for the known objects provide a slightly better agreement with the optical types than those obtained from the O8 spectral type set (see Table 3). The values of $Q$, corresponding to the G5 telluric standards, for each of our ISOCAM targets are listed in Table 4 . We also used the index $Q$ to derive spectral types for the three objects with no midIR excess in our sample. We obtained M1 $(Q=0.86)$ for 


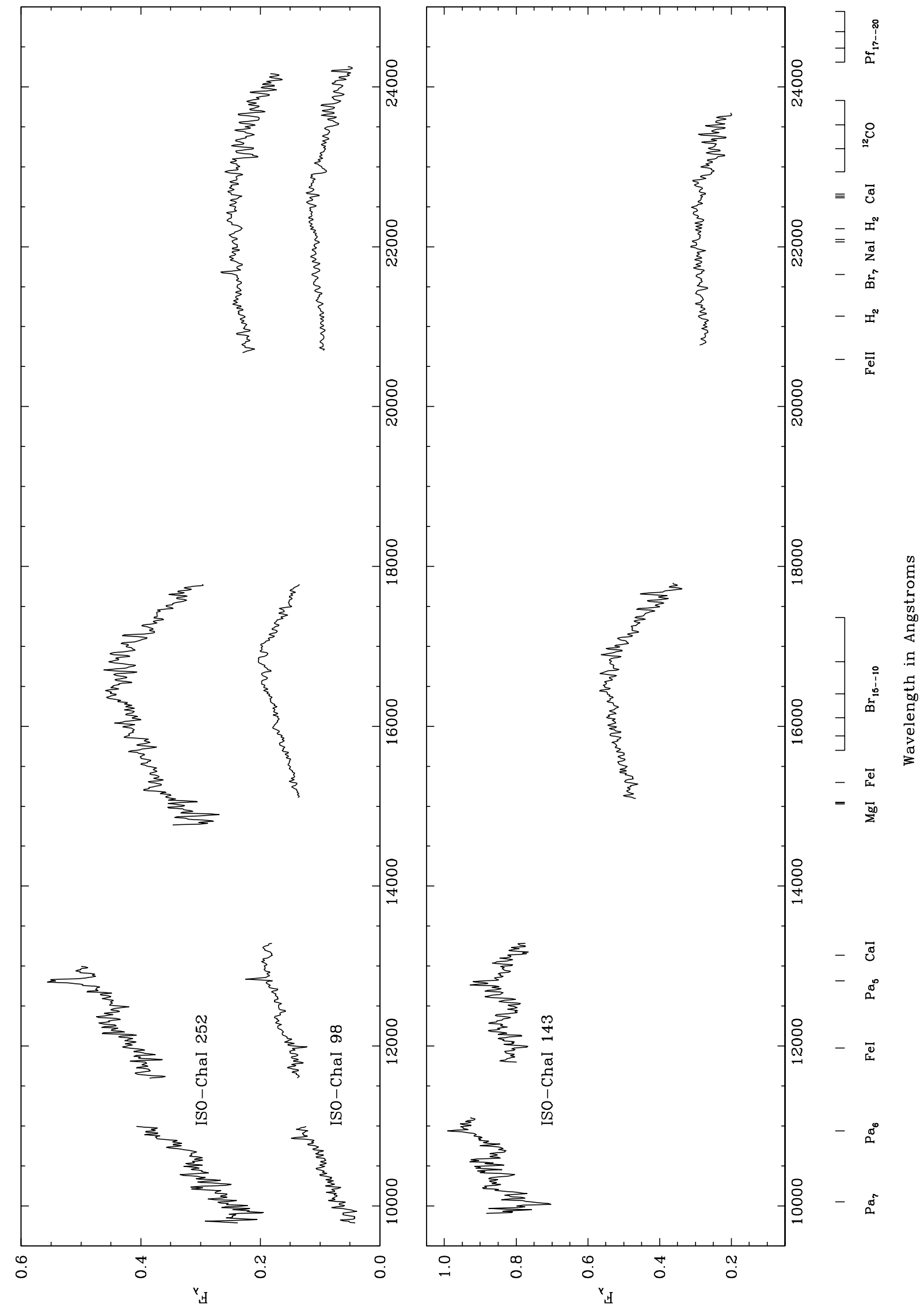

Fig. 4. JHK band spectra of ISO-ChaI 252, ISO-ChaI 98 and ISO-ChaI 143 in the Chamaeleon I dark cloud. The vertical scale $\left(F_{\lambda}\right)$ corresponding to ISO-ChaI 98 is shifted by -0.1 with respect to ISO-ChaI 252 . Prominent atomic and molecular features in the $0.98-2.45 \mu \mathrm{m}$ range are labeled. 
M. Gómez and P. Persi: ISO selected Cha I young stellar objects

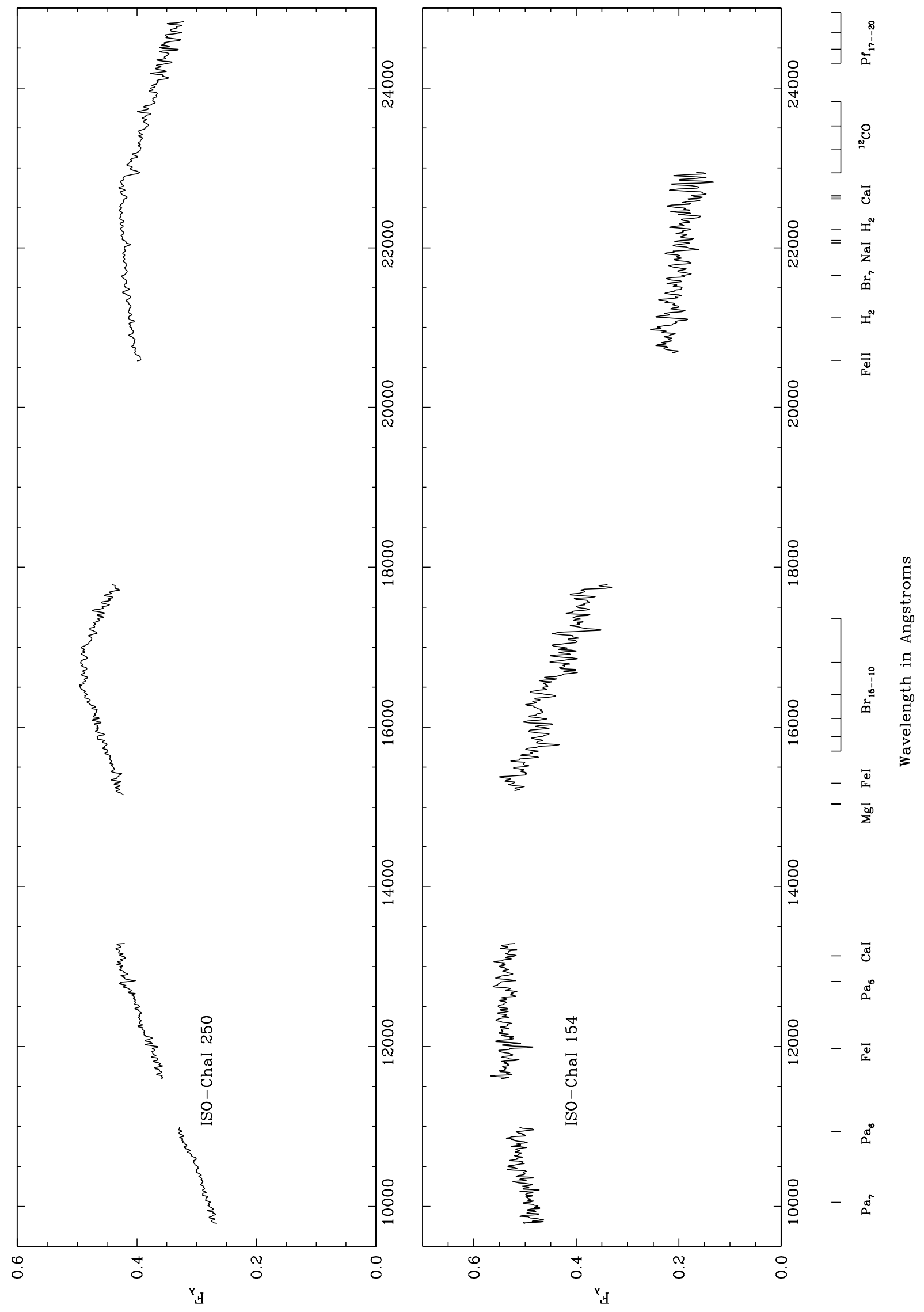

Fig. 5. JHK band spectra of ISO-ChaI 250, and ISO-ChaI 154 in the Chamaeleon I dark cloud. Prominent atomic and molecular features in the $0.98-2.45 \mu \mathrm{m}$ range are labeled. 


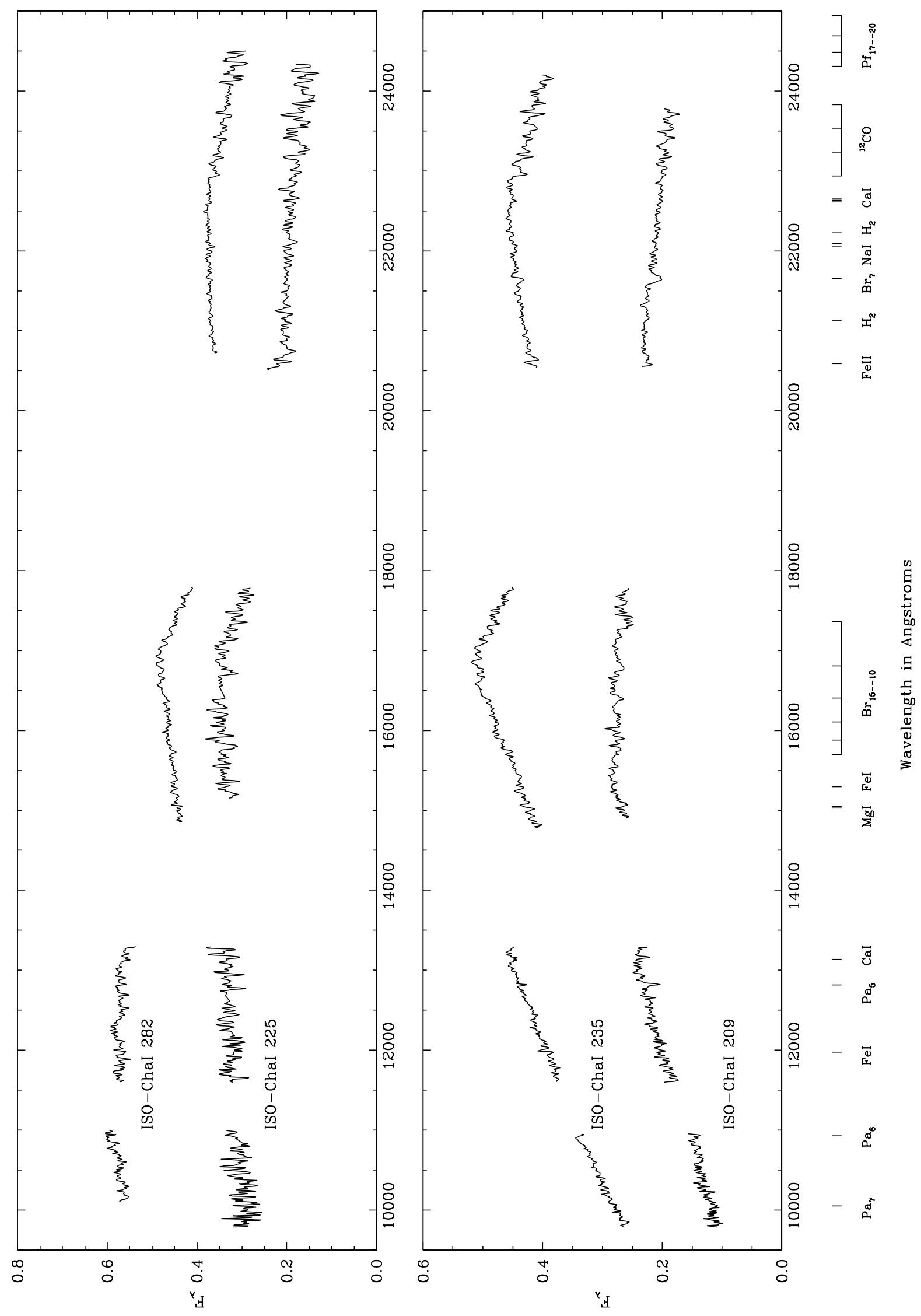

Fig. 6. JHK band spectra of ISO-ChaI 282, ISO-ChaI 225, ISO-ChaI-235 and ISO-ChaI 209 in the Chamaeleon I dark cloud. The vertical scale $\left(F_{\lambda}\right)$ corresponding ISO-ChaI 282 (upper panel) is offset by 0.15 with respect to ISO-ChaI 225 . ISO-ChaI-235 (lower panel) is shifted by 0.2 in relation to ISO-ChaI 209. Prominent atomic and molecular features in the $0.98-2.45 \mu \mathrm{m}$ range are labeled. 


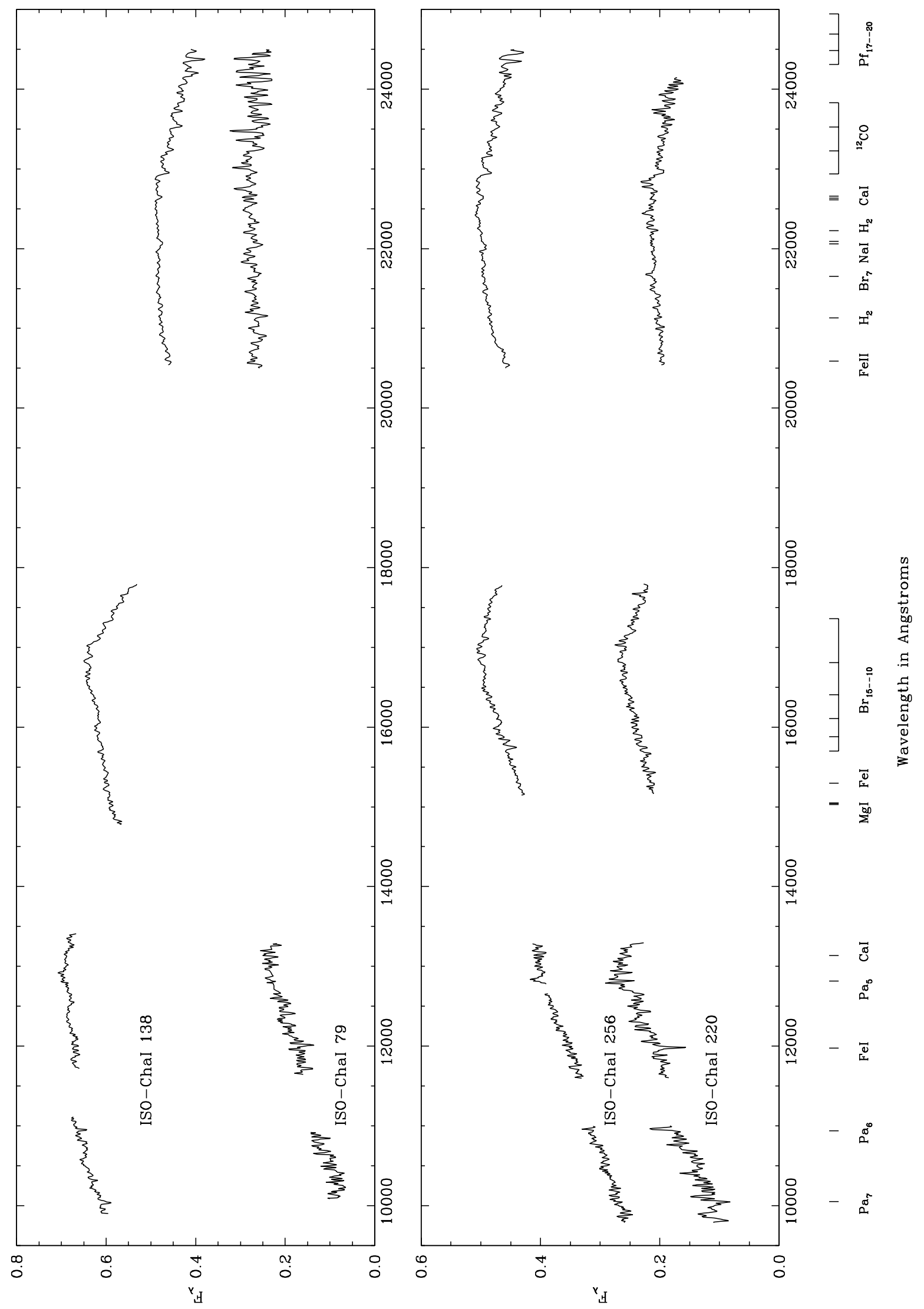

Fig. 7. $J H K$ band spectra of ISO-ChaI 138, ISO-ChaI 79, ISO-ChaI 256, and ISO-ChaI 220 in the Chamaeleon I dark cloud. The vertical scale $\left(F_{\lambda}\right)$ corresponding to ISO-ChaI 138 (upper panel) is offset by 0.3 with respect to ISO-ChaI 79 . ISO-ChaI 256 (lower panel) is shifted by 0.25 with respect to ISO-ChaI 220. Prominent atomic and molecular features in the $0.98-2.45 \mu \mathrm{m}$ range are labeled. 
Table 4. Spectral type, veilings, and masses of the ISO candidates.

\begin{tabular}{lccccc}
\hline \hline Name & $Q$ & Spectral Type $^{*}$ & $r_{\mathrm{K}}$ & $T_{\text {eff }}{ }^{* *}$ & $M / M_{\odot}$ \\
\hline ISO-ChaI 79 & 0.76 & M3 & +0.36 & 3260 & 0.22 \\
ISO-ChaI 95 & 0.51 & M8(M6-7) & -0.07 & 2430 & 0.025 \\
ISO-ChaI 98 & 0.65 & M5 & -0.14 & 2928 & 0.14 \\
ISO-ChaI 111 & 0.62 & M6(M5) & -0.13 & 2762 & 0.09 \\
ISO-ChaI 138 & 0.63 & M5.5 & -0.24 & 2845 & 0.09 \\
ISO-ChaI 143 & 0.61 & M6.5(M5.5) & -0.16 & 2679 & 0.085 \\
ISO-ChaI 154 & 0.92 & M0 & +0.03 & 3850 & 0.60 \\
ISO-ChaI 209 & 0.90 & M1 & +0.21 & 3720 & 0.50 \\
ISO-ChaI 220 & 0.69 & M4.5 & -0.00 & 3011 & 0.12 \\
ISO-ChaI 224 & 0.92 & M0 & +0.19 & 3850 & 0.45 \\
ISO-ChaI 225 & 0.83 & M2 & +0.68 & 3426 & 0.30 \\
ISO-ChaI 235 & 0.62 & M6(M5) & -0.09 & 2762 & 0.09 \\
ISO-ChaI 250 & 0.62 & M6(M5) & -0.18 & 2762 & 0.09 \\
ISO-ChaI 252 & 0.82 & M2 & -0.06 & 3426 & 0.30 \\
ISO-ChaI 256 & 0.65 & M5 & -0.04 & 2928 & 0.12 \\
ISO-ChaI 282 & 0.73 & M3.5 & +0.07 & 3177 & 0.19 \\
\hline
\end{tabular}

*Note: Spectral types between brackets are corrected by the different water absorption intensity between pre-main sequence and main sequence stars of the same $T_{\text {eff }}$, as estimated by Luhman \& Rieke (1999) for spectral types later than M6.

${ }^{* *}$ Note: We adopt Wilking et al. (1999) $T_{\text {eff }}$ calibration that comprises $\mathrm{M}$ sub-types later than M2. To assign $T_{\text {eff }}$ to the three objects (ISO-ChaI 154, ISO-ChaI 209, and ISO-ChaI 224) in this table with spectral types ealier than M2 we use Kenyon \& Hartmann (1995) temperature calibration.

ISO-ChaI $158, \mathrm{M} 5(Q=0.66)$ for ISO-ChaI 238 and M0 $(Q=0.93)$ for ISO-ChaI 239 .

Comerón et al. (2000) proposed a second reddeningfree $\mathrm{H}_{2} \mathrm{O}$ vapor index, $I_{\mathrm{H}_{2} \mathrm{O}}$, that compares the intensity of the absorption bands near $1.9 \mu \mathrm{m}$. This index is defined by the relative fluxes in four narrow band filters $(0.05 \mu \mathrm{m}$ width) centered at $1.675 \mu \mathrm{m}, 1.750 \mu \mathrm{m}, 2.075 \mu \mathrm{m}$, and $2.25 \mu \mathrm{m}$, lying in regions of reasonably good atmospheric transmission. On the contrary, Wilking et al. (1999) index $Q$ comprises regions close to the limit of the $K$-band window and thus may be affected by a poor telluric correction of the data. Assuming the Rieke \& Lebofsky (1985) reddening law over the $H K$ band $\left(\begin{array}{lll}A_{\lambda} & \propto \lambda^{-1.47}\end{array}\right)$ and denoting the fluxes at each narrow band filter by $f 1, f 2$, $f 3$, and $f 4$, respectively, the index $I_{\mathrm{H}_{2} \mathrm{O}}$ is expressed by:

$I_{\mathrm{H}_{2} \mathrm{O}}=(f 1 / f 2)(f 4 / f 3)^{0.76}$.

We calculated this index for the all the stars observed, including the three previously known T Tauri stars (Sz 84, $\mathrm{Sz}$ 97, and Sz 105) in the Lupus star-forming region and 18 of the ISOCAM sources. We excluded ISO-ChaI 79 as no reliable spectrum was obtained for this object around the wavelengths of these narrow band filters (see Fig. 7). Figure 8 shows the plot of the index $I_{\mathrm{H}_{2} \mathrm{O}}$ versus the M sub-types for these objects, derived from the index $Q$ (see

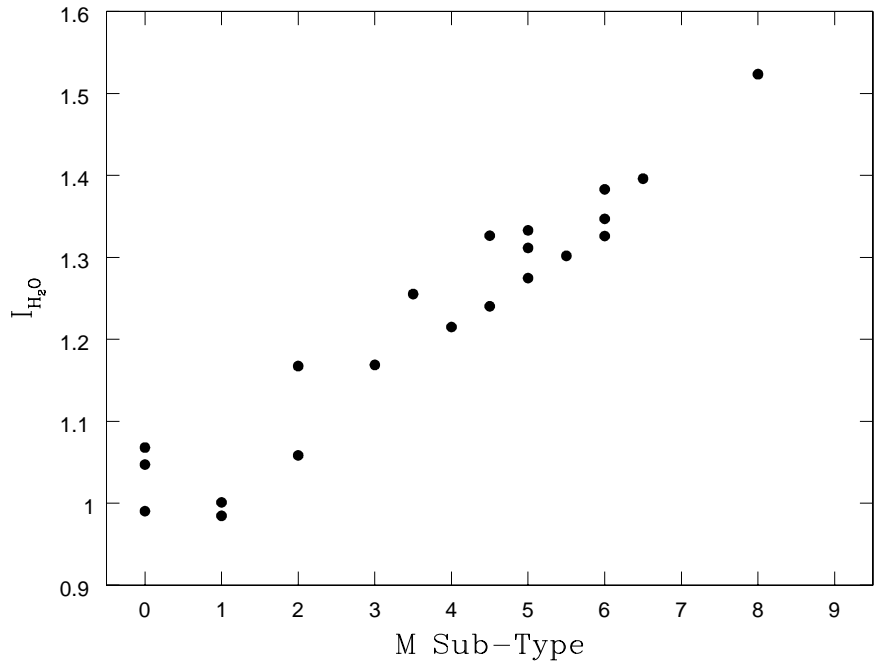

Fig. 8. The $1.9 \mu \mathrm{m}$ water vapor index, $I_{\mathrm{H}_{2} \mathrm{O}}$, defined by Comerón et al. (2000) versus the M sub-types for all the stars observed with SOFI. The spectral types are those obtained from the index $Q$, using the G5 spectral type stars as telluric corrector, listed in Tables 3 and 4. ISO-ChaI 79 was not included in this figure as no reliable spectrum was obtained around these wavelengths for this object (see Fig. 7).

Tables 3 and 4). A systematic trend is evident in the figure, although the correlation is rather poor. This trend gives some additional confidence to our spectral type estimations based on the index $Q$ for the newly detected ISOCAM sources.

We used the 6 objects in Table 3 and the $1.9 \mu \mathrm{m}$ water vapor index, $I_{\mathrm{H}_{2} \mathrm{O}}$, to obtain the following linear leastsquares fit:

$M$ sub_type $=(-15.9 \pm 3.8)+(16.0 \pm 3.0) \times I_{\mathrm{H}_{2} \mathrm{O}}$,

with a correlation coefficient $r=0.94$. We estimate an uncertainty of about 3 sub-classes in this calibration, considering typical errors in our measurements of the index $I_{\mathrm{H}_{2} \mathrm{O}}$ and the correlation coefficient of Eq. (3). This uncertainty is larger than that corresponding to the index $Q$ calibration ( 1.5 sub-classes). Relation 3 was derived from a the relatively small number of observed stars with optically known spectral types. However this calibration allows us an initial confrontation between the water vapor indexes.

In Fig. 9 we compare spectral types derived from both the indexes $Q$ and $I_{\mathrm{H}_{2} \mathrm{O}}$. Spectral types for the ISOCAM selected sources reported in this paper agree within their relatively large uncertainties. We caution, however, that additional observations are necessary to determine Eq. (3) more precisely before a complete agreement between the two procedures can be claimed.

Luhman \& Rieke (1999) and Comerón et al. (2000) have suggested that the water vapor indexes may saturate for stars as earlier as M6, losing sensitivity toward the latest M sub-types. The uncertainties in our index $I_{\mathrm{H}_{2} \mathrm{O}}$ determination precludes us to distinguish this effect. A more accurate treatment is required to estimate if the 


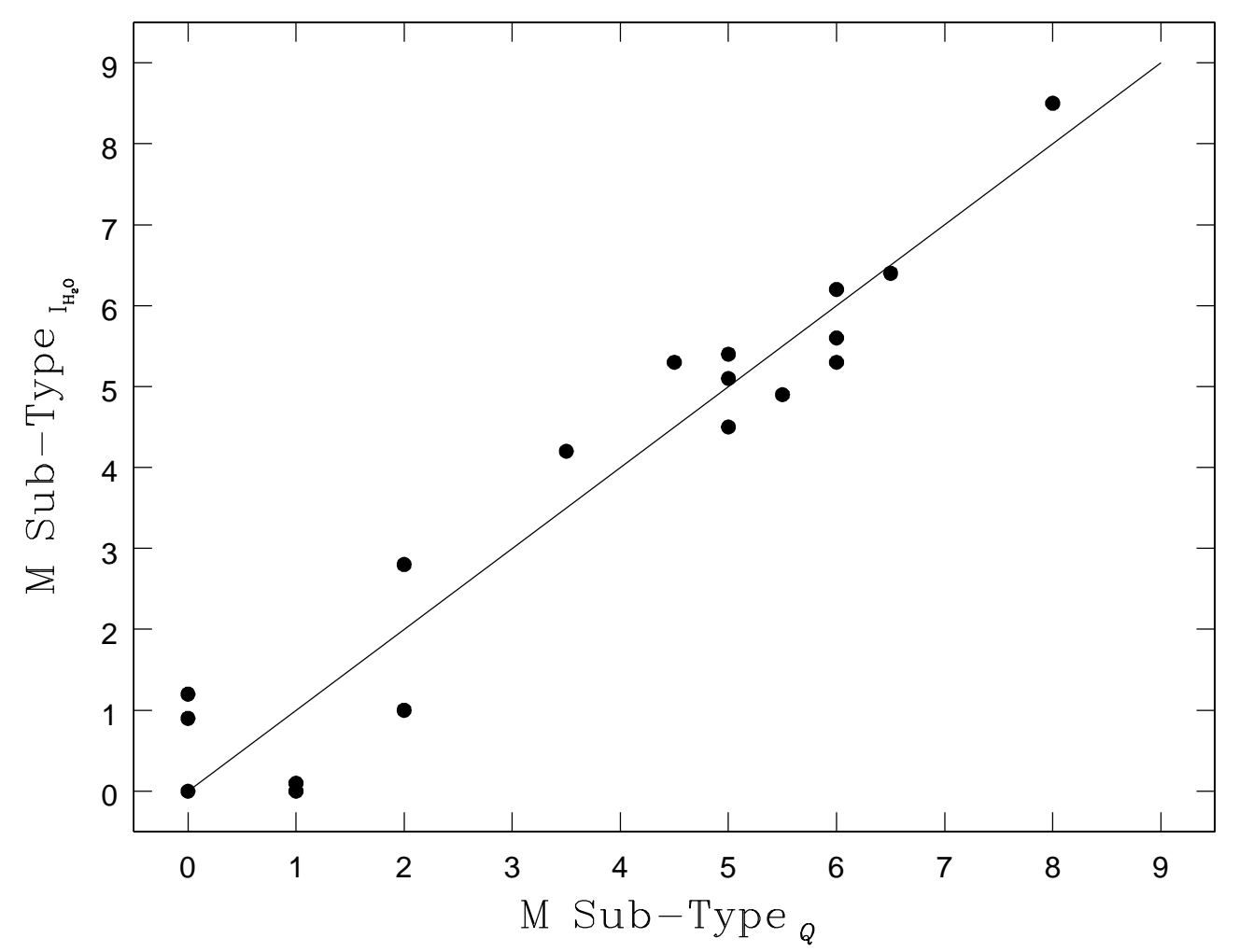

Fig. 9. Comparison between spectral types derived from the indexes $I_{\mathrm{H}_{2} \mathrm{O}}$ (Comerón et al. 2000) and $Q$ (Wilking et al. 1999) for objects in Table 1. The solid line corresponds to a linear relation with slope of 1 , shown as reference.

onset of water vapor saturation can significantly alter the spectral type determinations.

\subsubsection{Effects of veiling}

As noticed by Wilking et al. (1999) and Cushing et al. (2000) the infrared excess produced by the thermal emission from warm dust grains in circumstellar disks around young stellar objects may alter the index $Q$ and thus the spectral type estimations of our targets. To determine the $Q$ versus M sub-type relation these authors have used main sequence stars of optically known M sub-types, essentially devoted of any significant amount of infrared excesses.

This infrared excess emission can be characterized by $r_{\lambda}=F_{\lambda}^{\text {exc }} / F_{\lambda}^{\text {phot }}$, where $F_{\lambda}^{\text {exc }}$ is the measured flux, including the excess (circumstellar) emission, and $F_{\lambda}^{\text {phot }}$ is the flux due to the underlying stellar photosphere. Wilking et al. (1999) have simulated the spectra expected from young stars surrounding by circumstellar matter, adding the disk contribution to the spectra of known standard stars. These authors have then compared the index $Q$ calculated for stars of known spectral types with and without the disk emission. The spectral types obtained from star+disk systems that have veiling effects of $r_{\lambda} \sim 0.2$ are about 1 sub-class earlier than those derived for objects without the disk contribution. This effect increases with $r_{\lambda}$ and can account for $\sim 3$ sub-classes earlier for $r_{\lambda} \sim 0.6$.
In Table 4 we give $r_{K}$, the $K$-band excess, estimated for our target objects using the following relation,

$$
\begin{aligned}
E(H-K) & =(H-K)-(H-K)^{\text {phot }}-0.077 A_{V} \\
& =-2.5 \log \left[1 /\left(1+r_{K}\right)\right]
\end{aligned}
$$

derived by Meyer et al. (1997) (see also Wilking et al. 1999; Cushing et al. 2000). In this expression $(H-K)$ is the observed color (see Table 1$)$ and $(H-K)^{\text {phot }}$ corresponds to the intrinsic or photospheric color of each target. We have adopted the calibration from Wilking et al. (1999) to obtain intrinsic colors for our targets. Only for three objects in Table 4 with spectral types earlier than M2 (ISOChaI 154, ISO-ChaI 209, and ISO-ChaI 225) we have obtained intrinsic colors from Kenyon \& Hartmann (1995) as the Wilking et al. (1999) calibration only comprises M2-9 stars. Both color calibrations agree well in the common range. The third term in Eq. (4) corresponds to the excess in color due only to the extinction to each individual target. $A_{V}$ was derived from $A_{J}$ in Table 1 according to Rieke \& Lebofsky (1985) redding law $\left(A_{J}=0.28 A_{V}\right)$. As noticed by Wilking et al. (1999) and Cushing et al. (2000) this estimation provides only a lower limit to the veiling as Eq. (4) assumes $r_{H}=r_{J}=0$ (see also Meyer et al. 1997).

The ISOCAM targets in Table 4 have $r_{K}<0.2$ with exception of ISO-ChaI 79 and ISO-ChaI 225. We then expect our spectral type determinations using the $Q$ index be little affected by the effects of veiling except for these two objects (ISO-ChaI 79 and ISO-ChaI 225), that may 
actually have spectral types of $\sim 2-3$ sub-types later than those given in Table 4.

\subsubsection{Surface gravity effects}

Pre-main sequence stars are known to have surface gravities intermediate between those of dwarfs - luminosity class V - and giants - luminosity class III - (e.g., Greene \& Meyer 1995; Greene \& Lada 1996). However we have determined spectral types for our ISOCAM targets using the $Q$ index versus the $\mathrm{M}$ spectral type relation derived for M type main sequence standards (Wilking et al. 1999; Cushing et al. 2000). The water vapor telluric absorption in the $2 \mu \mathrm{m}$ spectral region is weaker in late-type giants than in dwarfs of the same spectral type (e.g., Kleinmann \& Hall 1986). For spectral types later than M6 the water vapor absorption bands tend to be similar in depth both for dwarfs and giants and then the spectral classification would result quasi independent of the surface gravity. Wilking et al. (1999) estimated a systematic error of about 1-2 sub-classes earlier than appropriate for stars earlier than M6 by assuming dwarfs rather than giants gravities.

For pre-main sequence stars Luhman \& Rieke (1999) have found, however, that the water absorption band is stronger than for main sequence stars of the same $T_{\text {eff }}$. These authors have also found that spectral types derived from the $Q$ index are $\sim 1$ sub-class later for M6-M7 and $\sim 1-2$ sub-classes for $>$ M 8 stars. This estimation is based on a comparison between spectral types derived using the $Q$ index and optically determined spectral types. We adopt this correction for the 5 objects in Table 4 with spectral types later than M6. We indicate the corrected spectral types between brackets in this table.

Lacking of a more appropriate calibration to estimate spectral types for potential young stars, we estimate a typical uncertainty of about 1-2 sub-classes in our spectral classification. This uncertainty is comparable to the expected precision for the $Q$ index versus M-sub type relation as mention in Sect. 3.3.1 (see also Wilking et al. 1999).

\subsubsection{Effective temperatures and masses}

To derive effective temperatures for our targets we have considered and compared three calibrations from the literature: a) Kenyon \& Hartmann (1995), b) Wilking et al. (1999), and c) Luhman (1999). Both Kenyon \& Hartmann (1995) and Wilking et al. (1999) temperature scales are obtained using M dwarf stars. Kenyon \& Hartmann (1995) calibration, combined with the Briceño et al. (1998) determination, extends from M0 to M8 spectral types, while Wilking et al. (1999) comprises M2-9 stars. The third scale, Luhman (1999), provides an intermediate scale between the luminosity class V and III objects, from M1 to M9.
The Kenyon \& Hartmann (1995) calibration is based on the Schmidt-Kaler (1982) work. More recently Briceño et al. (1998) used Henry et al. (1994) and Kirkpatrick et al. $(1993,1995)$ observations to test and continue this scale toward the latest M sub-types. The Wilking et al. (1999) calibration corresponds to a linear least-squares fit to a single data set (Jones et al. 1996). Luhman (1999) used Leggett et al. (1996) data to obtain a temperature scale for dwarf stars. The model atmosphere of Kirkpatrick et al. (1993) allows Luhman (1999) to extrapolate the calibration from M6 to M9 (see also Luhman \& Rieke 1998). The temperature scale for the giants was derived from different data sets. In particular, van Belle et al. (1999) spectra were used to derive the fit for spectral types earlier than M7 and Perrin et al. (1998) and Richichi et al. (1998) works for giants in the M7-M9 spectral range. Using these two independent calibrations, Luhman (1999) derived an intermediate scale between the dwarfs and giants.

Pre-main sequence objects have surface gravities intermediate between the luminosity class V and III stars and thus Luhman (1999) calibration, in principle, seems to be the most appropriate. The numerical values of this calibration are intermediate between those of Kenyon \& Hartmann (1995) and Wilking et al. (1999) and, in general, closer to Kenyon \& Hartmann (1995)'s temperatures.

The application of Luhman (1999) and Kenyon \& Hartmann (1995) spectral types $-T_{\text {eff }}$ conversion scales leads to results that are hard to conciliate with previous determinations for the high mass members of the Chamaeleon I cloud. In particular some of the stars with large near-IR excesses, when applying these calibrations, have ages too old $\left(\sim 5 \times 10^{7} \mathrm{yr}\right)$ in relation to the $\sim 3-5 \times$ $10^{6} \mathrm{yr}$ determined for the higher mass members of the cloud by Lawson et al. (1996). On the contrary, Wilking et al. (1999)'s calibration provides consistent results, in good concordance with previous works. In addition this calibration is based on an homogeneous set of standards whereas the other two involve combinations of different data. Luhman (1999) calibration used Kirkpatrick et al. (1993)'s model to extrapolate to the latest M sub-types. Model atmospheres of $\mathrm{M}$ dwarfs reasonably fit the overall shape of the observational data. However, several difficulties in fitting more specific features still remain (see, for example, Kirkpatrick et al. 1993; Allard et al. 1995). Thus we adopt the Wilking et al. (1999)'s calibration to place our targets on the HR diagram as it gives the clearest results, based on an uniform scale. Only for three objects in Table 4 with spectral types earlier than M2 (ISO-ChaI 154, ISO-ChaI 209, ISO-ChaI 224) we use the Kenyon \& Hartmann (1995) temperature scale.

Figure 10 shows the positions of the ISOCAM detected stars in the HR diagram. Luminosities have been obtained from Persi et al. (2000) (see also, Table 1). These authors have computed stellar (not bolometric) luminosities for the newly and previously known members of the Chamaeleon I cloud and compared their luminosities determinations for the higher mass pre-main sequence stars with those of Lawson et al. (1996). They have found 
a reasonable agreement within a factor of 2. Premain sequence evolutionary tracks (continuous lines) and isocrones (dash lines) are from D'Antona \& Mazzitelli $(1998)^{5}$.

Several evolutionary tracks (e.g., Burrows et al. 1997; Baraffe et al. 1998; Palla \& Stahler 1999) that are available, in principle, can be used to infer masses and ages. We have chosen D'Antona \& Mazzitelli (1998) model as these tracks cover a wide range of masses, from $0.017 M_{\odot}$ to $0.9 M_{\odot}$. Thus, they allow us to estimate masses and ages for all of our targets in a homogeneous manner. In addition this set of evolutionary tracks and isocrones provide consistent and plausible results for our group of targets, in reasonable agreement with the higher mass members of the cloud. Baraffe et al. (1998) and Palla \& Stahler (1999) tracks do not comprise sub-stellar objects. Burrows et al. (1997) model, on the contrary, provides detailed calculations in the sub-stellar regime extending up to $0.2 M_{\odot}$. The masses and ages derived from different tracks and isocrones, in the common mass range and for a given $T_{\text {eff }}$ calibration, typically agree within a factor of $<2$ in mass and $<3$ in age. This, however, introduces some uncertainty in the stellar/sub-stellar nature of some of the ISOCAM detected objects analyzed here. Table 4 lists masses for our targets derived from D'Antona \& Mazzitelli's tracks.

The ISOCAM detected sources have sub-solar masses down to the $\mathrm{H}$ burning limit or even, in the case of ISOChaI 95 (Cha H $\alpha$ 1), below this limit. Three of the stars in Table 4 have previous estimations of masses. For Sz 33 (ISO-ChaI 224) Lawson et al. (1996) derived masses of $0.3 M_{\odot}$ and $0.4 M_{\odot}$, from D'Antona \& Mazzitelli (1994) and Swenson et al. (1996) evolutionary tracks, respectively. Our mass determination, based on the Kenyon \& Hartmann (1995) temperature scale, agrees well with Lawson et al. (1996)'s estimation.

Comerón et al. (2000) estimated masses of $0.04-$ $0.05 M_{\odot}$ and $0.07-0.08 M_{\odot}$ for Cha $\mathrm{H} \alpha 1$ (ISO-ChaI 95) and Cha $\mathrm{H} \alpha 2$ (ISO-ChaI 111), respectively, using Baraffe et al. (1998) and Burrows et al. (1997) evolutionary tracks. Cha $\mathrm{H} \alpha 1$ is considered a bona-fide young brown dwarf member of the cloud. Cha $\mathrm{H} \alpha 2$ is a transition (substellar/stellar) object with a mass close to the H-burning limit. Considering typically errors in the mass determinations (roughly a factor of 2, at this low limit) our estimations are consistent with the sub-stellar or quasi substellar nature of these objects and agree well with previous works.

These targets span a range of ages from $1-3 \times 10^{5} \mathrm{yr}$ to $\sim 10^{7}$ yr. Only three objects (ISO-ChaI 225 , ISO-ChaI 209, and ISO-ChaI 154) in Fig. 9 have ages $>$ few $\times 10^{7}$ yr. The median age of the rest of the newly detected sources, is $\sim$ few $\times 10^{6} \mathrm{yr}$. The range and median age of these stars roughly correspond to the median age and to the spread in age found by Lawson et al. (1996) for the higher mass members of this cloud.

\footnotetext{
5 Available at http://www.mporzio.astro.it/ dantona/ prems.html.
}

Due to the high veiling of ISO-ChaI $225\left(r_{K} \sim+0.68\right)$ we may have underestimated the spectral type of this star in about 3 sub-type (see Sect. 3.3.2). Adopting a $T_{\text {eff }} \sim 400 \mathrm{~K}$ cooler, this star shifts to the right on the HR diagram, corresponding to decreasing values in both masses and ages $\left(0.1 M_{\odot}\right.$ and $5 \times 10^{6} \mathrm{yr}$, respectively). In Fig. 10 we have indicated with arrows the displacements that ISO-ChaI 225 as well as ISO-ChaI $79\left(r_{K} \sim+0.36\right)$ experiment correcting the spectral types of these objects for their high veiling values, according to Wilking et al. (1999)'s estimation. In this case, ISO-ChaI 79 practically coincides with the position of ISO-ChaI $98\left(0.14 M_{\odot}\right.$ and $\left.1-2 \times 10^{6} \mathrm{yr}\right)$ on this diagram.

For ISO-ChaI 209 and ISO-ChaI 154 (spectral types M1 and M0, respectively) we have used Kenyon \& Hartmann (1995) $T_{\text {eff }}$ calibration, as Wilking et al. (1999) relation comprises only spectral types later than M2. The temperatures derived by Wilking et al. (1999) are roughly $300 \mathrm{~K}$ cooler than those obtained by Kenyon \& Hartmann (1995), in the common range. If we adopt a correction of this amount, these targets shift to the right on the HR diagram, corresponding to decreasing masses and ages $\left(0.19 M_{\odot}\right.$ and $1 \times 10^{7} \mathrm{yr}$, for ISO-ChaI $209 ; 0.4 M_{\odot}$ and $3 \times 10^{7} \mathrm{yr}$, for ISO-ChaI 154), in better agreement with the other ISOCAM detected sources.

\subsection{Detection of emission features in the ISO observed sample}

The ISOCAM detected sample analyzed here corresponds to a quite homogeneous group. These thirteen sources have similar stellar luminosities and spectral indexes (see Table 1) and comparable spectral types and masses (see Table 4). However, within our resolution and sensitivity limit, we detected hydrogen emission lines in 5 of the 13 objects (i.e., $38 \%$ of the sample, see Table 2). These five ISOCAM objects are: ISO-ChaI 98, ISO-ChaI 143, ISOChaI 220, ISO-ChaI 252, and ISO-ChaI 256 (see Figs. 4 and 7 ). We find no correlation between the presence of $\mathrm{H}$ Paschen and Brackett lines in emission and the near-IR color excesses or the amount of veiling estimated for each target.

Najita et al. (1996) and Muzerolle et al. (1998) have investigated the formation of hydrogen recombination emission lines in magnetospheric zones of the CTTS. In this model the stellar magnetic field truncates the disk (at some inner radius) and the accretion continues onto the central object following the magnetic field lines. These authors suggest that, in general, emission lines are indicators of disk accretion (infall) rather than stellar wind (outflow). Thus the ISOCAM sources with $\mathrm{H}$ lines in emission are likely to be surrounding by circumstellar disks.

The homogeneity of the ISOCAM sample observed suggests that the lack of $\mathrm{H}$ emission lines detection may be due to a projection or geometrical effect. Sources with no emission lines are probably not favorably oriented (i.e., practically edge-on disks). Higher resolution and 


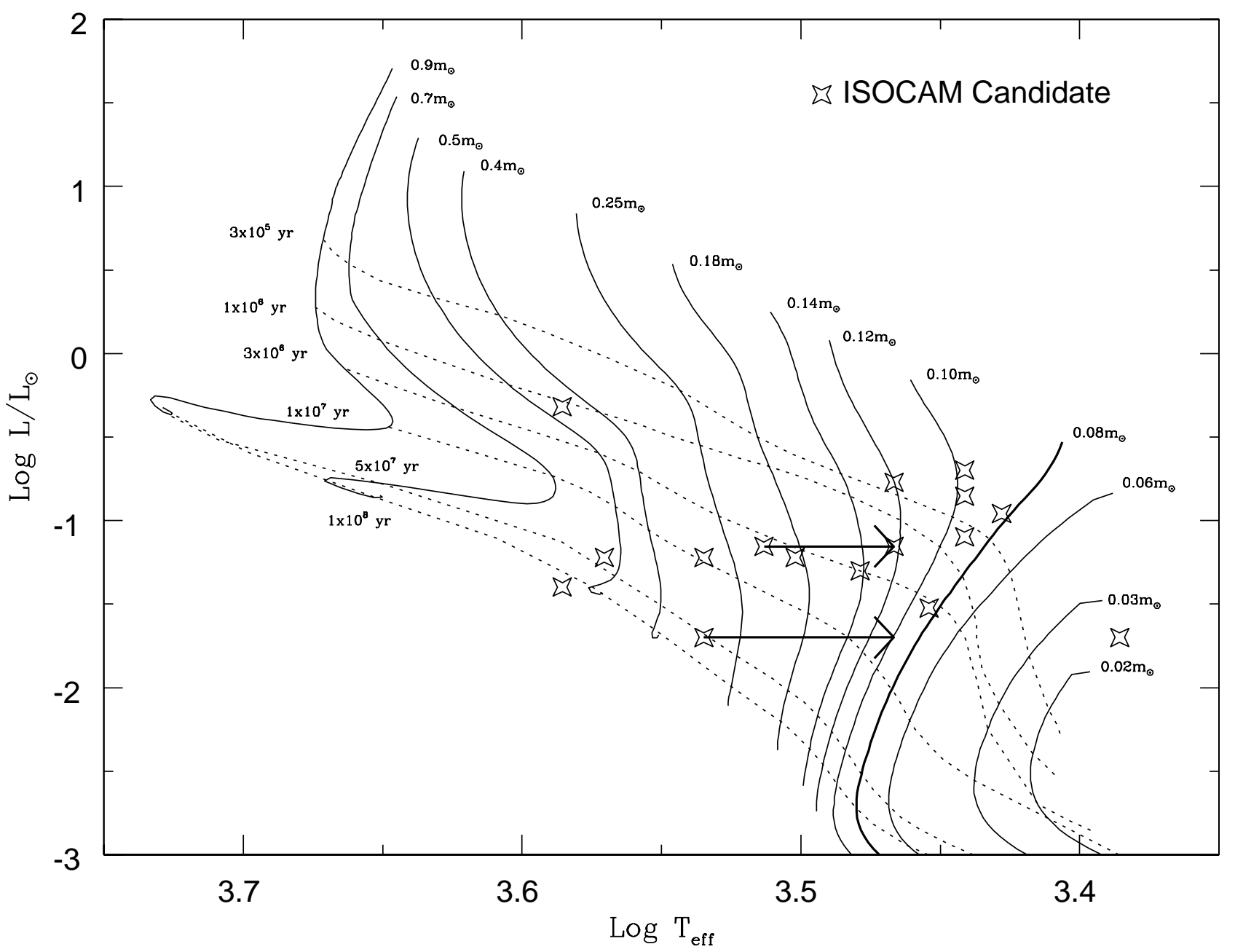

Fig. 10. HR diagram showing the positions of the ISOCAM selected low mass stars. Stellar (not bolometric) luminosities were taken from Persi et al. (2000) (see also, Table 1) and effective temperatures from Table 4. We have adopted the Wilking et al. (1999) temperature calibration in this paper that comprises M2 to M9 spectral types. For the three objects in Table 4 with spectral types M0-1 (ISO-ChaI 154, ISO-ChaI 209, and ISO-ChaI 224) we have used the Kenyon \& Hartmann (1995) temperature scale. Pre-main sequence evolutionary tracks, indicated with continuous lines, are from D'Antona \& Mazzitelli (1998). The thick continuous line corresponds to $0.08 M_{\odot}$, the $\mathrm{H}$ burning limit. The dashed lines correspond to the isocrones calculated also by the same authors. The arrows indicate the displacements on this diagram of ISO-ChaI 79 and ISO-ChaI 225 , when correcting the corresponding spectral types and effective temperatures due to the high values of veiling $\left(r_{K}=+0.36\right.$ and +0.68 , respectively) determined for these targets. Accordingly, the lengths of the arrows show displacements of 2 and 3 sub-types (see Sect. 3.3.2 and Wilking et al. 1999). The position of ISO-ChaI 79 roughly coincides with ISO-ChaI 98, in this case.

sensitivity data are required to properly test the effect of the disk inclination on the $\mathrm{H}$ emission lines detection.

\section{Summary and conclusions}

We obtained $0.95-2.5 \mu \mathrm{m}$ moderate $(R \sim 500)$ resolution spectra of 19 ISOCAM detected sources in the Chamaeleon I dark cloud. Three of these objects (ISOChaI 158, ISO-ChaI 238, and ISO-ChaI 239), without near- and mid-IR excess, show no spectral characteristics typical of young stellar objects (such as veiling or emission lines) and are likely background $\mathrm{M}$ dwarfs.

Thirteen of the sources are candidate very low mass members of the cloud proposed by Persi et al. (2000) on basis of the mid-IR color excess. The spectra of twelve of these sources are relatively flat and featureless in this wavelength range. The overall shapes and slopes of these spectra are typical of Class II young stellar objects (Greene \& Lada 1996). Both atomic and molecular lines (when in absorption) are partially veiled (see Table 2), suggesting the presence of continuum emission from circumstellar dust. In addition some of the sources show Paschen and Brackett lines in emission, also indicating the presence of disks. Persi et al. (2000) had already classified these objects as potential Class II members of the Chamaeleon I dark cloud on basis of the infrared spectral index $\left(\alpha_{\mathrm{IR}}=\mathrm{d} \log \left(\lambda F_{\lambda} / \mathrm{d} \log (\lambda)\right)\right.$. These objects have $-1.6<\alpha_{\mathrm{IR}}<0.3$, typical of Class II objects (see Table 1 ), with exception of ISO-ChaI $250\left(\alpha_{\mathrm{IR}}=-1.9\right)$, classified as a ClassII-III object by Persi et al. (2000). ISO-ChaI 154 
is the only source with mid-IR excess that shows nearIR spectral features characteristic of Class III objects or WTTS (Greene \& Lada 1996).

Three additional sources ( $\mathrm{Sz} 33$, Cha $\mathrm{H} \alpha$ 1, and Cha $\mathrm{H} \alpha 2$ ) are previously known members of the cloud. In particular, Cha $\mathrm{H} \alpha 1$ and Cha $\mathrm{H} \alpha 2$ are a bona-fide young brown dwarf and a transition object (with a mass close to the H-burning limit) optically detected by Comerón et al. (1998). We find no substantial difference between the spectra of these sub-stellar or quasi sub-stellar objects and those of very low mass stars in our sample at the resolution of our data. We notice, however, that allowing for typical uncertainties of about a factor of 2 in our mass determinations at least some of our targets are probably transition objects that lie in the boundary region between the stellar and the sub-stellar regimes.

We apply the $2 \mu \mathrm{m}$ water vapor index defined by Wilking et al. (1999) to estimate spectral types for the 13 mid-IR excess sources observed. These stars have spectral types M0-8. We use Persi et al.'s stellar luminosity determinations, in combination with D'Antona \& Mazzitelli latest pre-main sequence evolutionary tracks, to estimate masses and ages. The ISOCAM detected mid-IR excess sources have sub-solar masses down to or, in the case of ISO-ChaI 95 (Cha $\mathrm{H} \alpha$ 1), even below the H-burning limit. These objects span a wide range of ages from 1$3 \times 10^{5} \mathrm{yr}$ to $\sim \times 10^{7} \mathrm{yr}$. The median age of the newly detected sources is $\sim$ few $\times 10^{6} \mathrm{yr}$. The range of ages as well as the media age agree well with those found by Lawson et al. (1996) for the higher mass members of this cloud.

ISO-ChaI 225 has a high veiling $\left(r_{K} \sim 0.68\right)$ and thus our spectral type determination may be underestimated up to about 3 sub-types. Assigning a spectral type 3 subtype later (M5 instead of M2), this object has an age consistent with the rest of the ISOCAM detected low mass members of the Chamaeleon I dark cloud. ISO-ChaI 209 and ISO-ChaI 154 have spectral types M1 and M0, respectively. We used Kenyon \& Hartmann (1995) calibration to assign $T_{\text {eff }}$ to these targets. This temperature scale is $\sim 300$ K hotter than Wilking et al. (1999)'s scale. Considering this correction both stars shift to the right on the HR diagram and have ages $\sim 1-3 \times 10^{7}$ yr, in reasonable agreement with the rest of the ISOCAM detected sources and higher mass members of the cloud.

We found that 12 of the 13 ISOCAM detected candidate members of the Chamaeleon I dark cloud show near-IR spectroscopic features typical of Class II objects. Additional spectroscopic observations are required to investigate the physical properties of the 21 remaining very low luminosity candidate members of the cloud proposed by Persi et al. (2000). This group of objects may include about 19 stars with similar near-IR spectroscopic characteristics as Class II objects in view of the high detection rate of likely young members of the cloud found in this contribution. Near-IR spectroscopy is required to estimate masses for these 21 remaining objects. Combined mass determinations for all the ISOCAM detected objects would allow us to explore the behavior of the IMF of the cloud in the sub-stellar regime. This function is presently very poorly known for stars close and below the $\mathrm{H}$ burning limit.

Acknowledgements. We are grateful to the ESO staff for assistance during the observing run, specially to Leonardo Vanzi for technical support with SOFI. We thank the referee, Thomas Greene, for helpful criticisms and suggestions that improved the content and presentation of this paper. This research has made use of the SIMBAD database, operated at CDS, Strasbourg, France.

\section{References}

Allard, F., \& Hauschildt, P. H. 1995, ApJ, 445, 433

Baraffe, I., Chabrier, G., Allard, F., \& Hauschildt, P. H. 1998, A\&A, 337, 403

Bessell, M. S., \& Brett, J. M. 1988, PASP, 100, 1134

Briceño, C., Hartmann, L., Stauffer, J., \& Martín, E. 1998, AJ, 115,2074

Burrows, A., Marley, M., Hubbard, W. B., et al. 1997, ApJ, 491,856

Cambrésy, L., Copet, E., Epchtein, N., et al. 1998, A\&A, 338, 977

Carr, J. S. 1989, ApJ, 345, 522

Carr, J. S. 1990, AJ, 100, 1244

Casali, M. M., \& Eiroa, C. 1996, A\&A, 306, 427

Casali, M. M., \& Matthews, E. H. 1992, MNRAS, 258, 399

Comerón, F., Neuhäuser, R., \& Kaas, A. A. 2000, A\&A, 359, 269

Comerón, F., Rieke, G. H., \& Neuhäuser, R. 1998, A\&A, 343, 477

Cushing, M. C., Tokunaga, A. T., \& Kobayashi, N. 2000, AJ, 119,3019

D'Antona, F., \& Mazzitelli, I. 1994, ApJS, 90, 467

Feigelson, E. D., \& Kriss, G. A. 1989, ApJ, 338, 262

Feigelson, E. D., Casanova, S., Montmerle, T., \& Guibert, J. 1993, ApJ, 416, 623

Gauvin, L. S., \& Strom, K. M. 1992, ApJ, 385, 217

Greene, T. P., \& Lada, C. J. 1996, AJ, 112, 2184

Greene, T. P., \& Meyer, M. R. 1995, ApJ, 450, 233

Gómez, M., \& Kenyon, S. J. 2001, AJ, 121, 974

Henry, T. J., Kirkpatrick, J. D., \& Simons, D. A. 1994, AJ, 108,1437

Hughes, J., Hartigan, P., Krautter, J., \& Kelemen, J. 1994, AJ, 108, 1071

Johnson, H. L., \& Morgan, W. W. 1953, ApJ, 117, 313

Jones, H. R. A., Longmore, A. J., Allard, F., \& Hauschildt, P. H. 1996, MNRAS, 280, 77

Kenyon, S. J., \& Hartmann, L. 1995, ApJS, 101, 117

Kenyon, S. J., \& Gómez, M. 2001, AJ, 121, 2673

Kirkpatrick, J. D., Henry, T. J., \& Simons, D. A. 1995, AJ, 109, 797

Kirkpatrick, J. D., Kelly, D. J., Rieke, G. H., \& Liebert, J. 1993, ApJ, 402, 643

Kleinmann, S. G., \& Hall, D. N. B. 1986, ApJS, 62, 501

Lawson, W. A., Feigelson, E. D., \& Huenemoerder, D. P. 1996, MNRAS, 280, 1071

Leggett, S. K., Allard, F., Berriman, G., Dahn, C. C., \& Hauschildt, P. H. 1996, ApJS, 104, 117

Lidman, C., Cuby, J.-G., Vanzi, L., \& Hainaut, O. 2000, SOFI User's Manual, Doc. No. LSO-MAN-ESO-40100-0003, Issue 1.3 (available at: http://www.ls.eso.org/lasilla/ Telescopes/NEWNTT/sofi/manual/SOFImanual.html) 
Luhman, K. L. 1999, ApJ, 525, 466

Luhman, K. L., \& Rieke, G. H. 1998, ApJ, 497, 354

Luhman, K. L., \& Rieke, G. H. 1999, ApJ, 525, 440

Lucas, P. W., Roche, P. F., Allard, F., \& Hauschildt, P. H. 2001, MNRAS, 326, 695

Meyer, M. R., Calvet, N., \& Hillenbrand, L. A. 1997, AJ, 114, 288

Meyer, M. R., Edwards, S., Hinkle, K. H., \& Strom, S. 1998, ApJ, 508, 397

Muzerolle, J., Calvet, N., \& Hartmann, L. 1998, ApJ, 492, 743

Najita, J., Carr, J. S., \& Tokunaga, A. T. 1996, ApJ, 456, 292

Neuhäuser, R., \& Comerón, F. 1998, Science, 282, 83

Neuhäuser, R., \& Comerón, F. 1999, A\&A, 350, 612

Palla, F., \& Stahler, S. W. 1999, ApJ, 525, 772

Persi, P., Marenzi, A. R., Olofsson, G., et al. 2000, A\&A, 357, 219

Persi, P., Marenzi, A. R., Gómez, M., \& Olofsson, G. 2001, A\&A, 376, 907

Perrin, G., Coudé du Foresto, V., Ridgway, S. T., et al. 1998, A\&A, 331, 619
Pickles, A. J. 1998, PASP, 110, 863

Richichi, A., Ragland, S., Stecklum, B., \& Leinert, C. 1998, A\&A, 338, 527

Rieke, G. H., \& Lebofsky, M. J. 1985, AJ, 288, 618

Schmidt-Kaler, T. 1982, in Landolt-Börstein, Group VI, vol. 2, ed. K. H. Hellwege (Berlin: Springer), 454

Schwartz, R. D. 1977, ApJS, 35, 161

Strom, K. M., Strom, S. E., \& Merrill, K. M. 1993, ApJ, 412, 233

Suto, H., Mizutani, K., \& Toshinori, M. 1989, MNRAS, 239, 139

Swenson, F. J., Faulkner, J., Rogers, F. J., \& Iglesias, C. A. 1994, ApJ, 425, 286

van Belle, G. T., Lane, B. F., Thompson, R. R., et al. 1999, AJ, 117, 512

Wallace, L., \& Hinkle, K. 1997, ApJS, 111, 445

Wallace, L., Meyer, M. R., Hinkle, K., \& Edwards, S. 2000, ApJ, 535, 325

Wilking, B. A., Greene, T. P., \& Meyer, M. R. 1999, AJ, 117, 469 